MATHEMATICS OF COMPUTATION

Volume 71 , Number 238 , Pages $553-582$

S 0025-5718(01)01354-0

Article electronically published on November 28, 2001

\title{
LOCALIZATION EFFECTS AND MEASURE SOURCE TERMS IN NUMERICAL SCHEMES FOR BALANCE LAWS
}

\author{
LAURENT GOSSE
}

\begin{abstract}
This paper investigates the behavior of numerical schemes for nonlinear conservation laws with source terms. We concentrate on two significant examples: relaxation approximations and genuinely nonhomogeneous scalar laws. The main tool in our analysis is the extensive use of weak limits and nonconservative products which allow us to describe accurately the operations achieved in practice when using Riemann-based numerical schemes. Some illustrative and relevant computational results are provided.
\end{abstract}

\section{INTRODUCTION}

This paper deals with the numerical analysis of weak solutions to nonhomogeneous hyperbolic equations written in conservation form, 31. We will focus on two significant examples. The first one is the special case of relaxation approximation to the scalar conservation law (11)-(2), 28, 29. The second one corresponds to the general case of a scalar balance law (26). These two problems share the common feature of being solvable within the framework of BV functions and Kružkov's theory, 31 , 41.

In both cases, when designing any numerical scheme, one has to face the issue of choosing an efficient treatment of the zero-order term. The constraints are mostly stability in stiff regimes (as pointed out in, e.g., 18, 33) together with some consistency with an expected asymptotic behavior. For our examples, we distinguished between relaxation, for which the convergence towards the equilibrium manifold (the local Maxwellian) is the key point and the general balance law, where a longtime decay to some steady regime should occur in order to respect the dynamics of the differential underlying problem, 35]. It turns out that these two criteria are actually difficult to meet within the existing approaches, [7, 8, 13, 23.

One goal of the present article is to propose to work out both objectives efficiently in a simple self-contained Godunov approach. The basic trick is therefore to consider special Riemann problems endowed with some "measure-valued source terms" (see the systems (12) and (49)) which are to be processed carefully: consult Propositions 1 and 5. In return, this kind of formulation ensures that the asymptotic behavior will be respected under very reasonable stability restrictions. In $\S 2.6, \S 3.7$, we provide computational results on some classical problems in order to demonstrate that our numerical approaches are realizable and efficient.

Received by the editor December 13, 1999.

2000 Mathematics Subject Classification. Primary 65M06, 65M12; Secondary 35F25.

Key words and phrases. Balance laws, finite difference schemes, nonconservative products, relaxation schemes. 
Going a bit deeper into the contents of this work, we describe roughly the idea lying at the heart of the matter. For both the relaxing system and the scalar balance law, we consider a localization function depending either on the time or the space variable. This function is usually smooth at the level of the differential problem; this means that the relaxation or the reaction process is applied more or less uniformly in the domain. At the numerical level, this smoothness is not realistic any longer since it leads to intricate and computationally expensive schemes based mostly on generalized Riemann problems whose structure is highly more intricate compared to homogeneous ones, 15, 16. One is therefore led to tackle some singular localization functions, describing the fact that the relaxing or reactive processes are ignited and switched off suddently at particular moments or locations, as times increase. This actually matches the real structure of widely-used practical algorithms, including the classical time-splitting techniques or the so-called method of lines.

The mathematical tools required to carry out such a program have been recently developed since they go beyond classical distribution theory to consider ambiguous nonconservative products. Since weak solutions of hyperbolic equations are likely to be discontinuous, their product with Dirac measures is unstable and the relevant theory is developed in [5, 9, 12, 24, 32, 36. At the numerical level, the key point is to be able to compute accurately such measure-valued terms: some attempts have already been proposed in [17, 19, 20, 22, 23]; see also [8, 21, 40, 39] for closely related works. We stress that our results do not contradict [26].

The paper is organized as follows. In Section 2, we consider the relaxing approximations with a time-based localization function. We show compactness properties as this function concentrates. A Godunov scheme is naturally deduced for which it is possible to prove convergence towards the entropy solution in the infinite relaxation limit. In Section 3, a similar program is carried out for a general scalar balance law with a localization function depending on the space variable. In this last case, one has to face new issues as nonlinear resonance may occur, [27], which we shall systematically exclude with convenient hypotheses. In Section 4, we conclude and propose some perspectives for future developments.

\section{The SPECIAL CASE OF RELAXATION APPROXimations}

2.1. Motivation. We are interested in studying relaxation approximations in the sense of [28, 29] to the entropy solution of the scalar conservation law:

$$
\left\{\begin{array}{l}
\partial_{t} u+\partial_{x} f(u)=0 \\
u(., 0)=u_{0} \in B V(\mathbb{R}) .
\end{array}\right.
$$

Therefore, we can consider the following system:

$$
\left\{\begin{array}{l}
\partial_{t} u+\partial_{x} v=0 \\
\partial_{t} v+c^{2} \partial_{x} u=-\mu(v-f(u)) \\
u(., 0)=u_{0} \text { and } v(., 0)=f\left(u_{0}\right)
\end{array}\right.
$$

where the parameter $\mu>0$ is supposed to be very big and the velocity $c$ satisfies the classical subcharacteristic condition. The system (2) has been widely used as a building block inside relaxation schemes for (11) mainly because of its simple semilinear structure. Actually, as it is the common case for nonhomogeneous problems, the large majority of numerical algorithms actually does not solve (2). Most of them involve a free transport stage and a projection on the local equilibrium manifold at each time step $\Delta t>0$. So, it may be of interest to study a variant of 
(2) taking this observation into account. The relaxation term can be rewritten the following way:

$$
v-f(u)=(v-f(u)) \cdot 1=(v-f(u)) \partial_{t} b(t),
$$

together with the choice $b(t)=t$. This can be referred to as the continuous case. Consequently, in order to move towards a discrete one, we introduce $b^{\varepsilon}$ as a sequence of increasing Lipschitz continuous functions depending only on the time variable $t \in$ $\mathbb{R}^{+}$and we aim to investigate the behaviour of solutions to the following modified system:

$$
\left\{\begin{array}{l}
\partial_{t} u^{\varepsilon}+\partial_{x} v^{\varepsilon}=0 \\
\partial_{t} v^{\varepsilon}+c^{2} \partial_{x} u^{\varepsilon}=-\mu\left(v^{\varepsilon}-f\left(u^{\varepsilon}\right)\right) \partial_{t} b^{\varepsilon}(t) .
\end{array}\right.
$$

One interesting problem from the computational point of view is the limit when $b^{\varepsilon}$ becomes piecewise constant as $\varepsilon \rightarrow 0$. This corresponds to a relaxation mechanism which is ignited and switched off only at special instants $t^{n}=n \Delta t, n \in \mathbb{N}$, and matches the real nature of practical algorithms.

2.2. Introduction of a singular relaxation system. Let us begin with a remark which will be of constant use in the sequel: thanks to its linear convective part, the system (3) partly decouples, [1]:

$$
\left\{\begin{array}{l}
\partial_{t} w^{\varepsilon}+c \partial_{x} w^{\varepsilon}=\mu G\left(w^{\varepsilon}, z^{\varepsilon}\right) \partial_{t} b^{\varepsilon}(t) \\
\partial_{t} z^{\varepsilon}-c \partial_{x} z^{\varepsilon}=-\mu G\left(w^{\varepsilon}, z^{\varepsilon}\right) \partial_{t} b^{\varepsilon}(t)
\end{array}\right.
$$

where:

$$
w=-(v+c u) ; z=v-c u ; G(w, z)=\frac{1}{2}(-w+z)-f\left(-\frac{w+z}{2 c}\right) .
$$

Without any loss of generality, we can assume that $G(0,0)=0$. We suppose also that $f$ lies in $C^{2}(\mathbb{R})$. We now make the definition of $b^{\varepsilon}$ a bit more precise. Namely, relying on the choice of an arbitrary parameter $\Delta t>0$ and for any $n \in \mathbb{N}_{*}$, we select:

(6)

$$
b^{\varepsilon}(t)= \begin{cases}(n-1) \Delta t & (n-1) \Delta t \leq t<n \Delta t-\varepsilon, \\ \Delta t \cdot B\left(1+\frac{t-n \Delta t}{\varepsilon}\right)+(n-1) \Delta t & n \Delta t-\varepsilon \leq t<n \Delta t, \\ \text { with: } B \in C^{1}(\mathbb{R}) ; B(0)=0 ; B(1)=1 ; B^{\prime} \geq 0 .\end{cases}
$$

Consequently, there holds $\left(\mathbf{1}_{A}\right.$ stands for the characteristic function of the set $A$ ):

$$
\partial_{t} b^{\varepsilon}(t)=\frac{\Delta t}{\varepsilon} \sum_{n \in \mathbb{N}_{*}} B^{\prime}\left(1+\frac{t-n \Delta t}{\varepsilon}\right) \mathbf{1}_{t \in[n \Delta t-\varepsilon, n \Delta t[},
$$

a quantity which clearly converges in the weak topology of measures towards a Dirac comb as $\varepsilon \rightarrow 0$.

Lemma 1. Assume the initial data for (4) $\left(w_{0}, z_{0}\right)$ lie in $L^{1} \cap B V(\mathbb{R})$ and that the subcharacteristic condition $c \geq\left\|f^{\prime}\left(u^{\varepsilon}\right)\right\|_{L^{\infty}}$ holds; then, the sequence $\left(w^{\varepsilon}, z^{\varepsilon}\right)$ of solutions of (4), (5), (6) is relatively compact in the strong topology of $L_{\mathrm{loc}}^{1}(\mathbb{R} \times] 0, T[)$ as $\varepsilon \rightarrow 0$.

Proof. It relies mainly on the quasi-monotonicity property of $G$ together with standard BV compactness arguments. First, notice that because of the semilinearity of (4), the Cauchy problem admits a unique solution for $\varepsilon>0$ by the method of characteristics. We want now to derive estimates. 
- We multiply (4) by $\left(\operatorname{sgn}\left(w^{\varepsilon}\right), \operatorname{sgn}\left(z^{\varepsilon}\right)\right)$ and integrate on $x \in \mathbb{R}$ to get:

$$
\left\{\begin{array}{l}
\partial_{t} \int_{\mathbb{R}}\left|w^{\varepsilon}\right|(x, t) \cdot d x=\mu \int_{\mathbb{R}} \operatorname{sgn}\left(w^{\varepsilon}\right) G\left(w^{\varepsilon}, z^{\varepsilon}\right) \partial_{t} b^{\varepsilon}(t) \cdot d x, \\
\partial_{t} \int_{\mathbb{R}}\left|z^{\varepsilon}\right|(x, t) \cdot d x=-\mu \int_{\mathbb{R}} \operatorname{sgn}\left(z^{\varepsilon}\right) G\left(w^{\varepsilon}, z^{\varepsilon}\right) \partial_{t} b^{\varepsilon}(t) \cdot d x .
\end{array}\right.
$$

Since we assumed $G(0,0)=0$, we can use the mean-value theorem to get:

$$
G\left(w^{\varepsilon}, z^{\varepsilon}\right)=-\frac{w^{\varepsilon}}{2 c}\left(c-f^{\prime}(\xi)\right)+\frac{z^{\varepsilon}}{2 c}\left(c+f^{\prime}(\xi)\right) .
$$

This expresses that $G$ is decreasing with respect to its first variable, and increasing with respect to its second one; in particular, this implies the so-called quasi-monotonicity property, [1]. It remains to add the previous equations and to notice that $\operatorname{sgn}(x) . y \leq|y|$ for any $x, y$ in $\mathbb{R}$ in order to get:

$$
\partial_{t} \int_{\mathbb{R}}\left(\left|w^{\varepsilon}\right|+\left|z^{\varepsilon}\right|\right)(x, t) \cdot d x \leq 0 .
$$

- We differentiate (44) with respect to $x$, multiply the resulting equations by $\left(\operatorname{sgn}\left(\partial_{x} w^{\varepsilon}\right), \operatorname{sgn}\left(\partial_{x} z^{\varepsilon}\right)\right)$ and use exactly the same arguments to get:

$$
\partial_{t} \int_{\mathbb{R}}\left(\left|\partial_{x} w^{\varepsilon}\right|+\left|\partial_{x} z^{\varepsilon}\right|\right)(x, t) \cdot d x \leq 0
$$

This BV-bound also provides an $L^{\infty}$ one: this garantees that the subcharacteristic condition can be realized.

- We integrate (4) with respect to the time variable $t>t_{0}$ :

$$
\left\{\begin{aligned}
\int_{\mathbb{R}}\left|w^{\varepsilon}(x, t)-w^{\varepsilon}\left(x, t_{0}\right)\right| \cdot d x \leq & \int_{\mathbb{R}} \int_{t_{0}}^{t} c\left|\partial_{x} w^{\varepsilon}(x, s)\right| \\
& +\mu\left|G\left(w^{\varepsilon}, z^{\varepsilon}\right)(x, s)\right| \partial_{t} b^{\varepsilon}(s) . d s . d x \\
\int_{\mathbb{R}}\left|z^{\varepsilon}(x, t)-z^{\varepsilon}\left(x, t_{0}\right)\right| \cdot d x \leq & \int_{\mathbb{R}} \int_{t_{0}}^{t} c\left|\partial_{x} z^{\varepsilon}(x, s)\right| \\
& +\mu\left|G\left(w^{\varepsilon}, z^{\varepsilon}\right)(x, s)\right| \partial_{t} b^{\varepsilon}(s) . d s . d x .
\end{aligned}\right.
$$

We add both inequalities and use the mean-value theorem to get:

$$
\begin{aligned}
\int_{\mathbb{R}}\left|w^{\varepsilon}(x, t)-w^{\varepsilon}\left(x, t_{0}\right)\right|+\left|z^{\varepsilon}(x, t)-z^{\varepsilon}\left(x, t_{0}\right)\right| . d x \\
\leq\left|t-t_{0}\right|\left[c\left(T V\left(w_{0}\right)+T V\left(z_{0}\right)\right)\right] \\
\quad+2 \mu\left[\left\|w_{0}\right\|_{L^{1}(\mathbb{R})}+\left\|z_{0}\right\|_{L^{1}(\mathbb{R})}\right]\left(b^{\varepsilon}(t)-b^{\varepsilon}\left(t_{0}\right)\right),
\end{aligned}
$$

where $T V($.$) denotes the total variation in x$ of a given function. We notice that there is a global $\mathrm{BV}$-regularity on the sequence $Z^{\varepsilon} \stackrel{\text { def }}{=}\left(w^{\varepsilon}, z^{\varepsilon}\right)$ since:

$$
\begin{aligned}
& \sup _{h \neq 0} \int_{[0, T] \times \mathbb{R}} \frac{\left|Z^{\varepsilon}(x+h, t)-Z^{\varepsilon}(x, t)\right|}{h}+\frac{\left|Z^{\varepsilon}(x, t+h)-Z^{\varepsilon}(x, t)\right|}{h} \cdot d x . d t \\
& \quad \leq T\left[(1+c)\left(T V\left(w_{0}\right)+T V\left(z_{0}\right)\right)+2 \mu\left(\left\|w_{0}\right\|_{L^{1}(\mathbb{R})}+\left\|z_{0}\right\|_{L^{1}(\mathbb{R})}\right)\right] .
\end{aligned}
$$

This means that $\left(w^{\varepsilon}, z^{\varepsilon}\right) \in B V(\mathbb{R} \times[0, T])$.

As a consequence of the classical Helly's compactness theorem, the sequence $\left(w^{\varepsilon}, z^{\varepsilon}\right)$ is relatively compact in $L_{\text {loc }}^{1}(\mathbb{R} \times] 0, T[)$. 
2.3. A meaning for the ambiguous product. We now want to shed some light on the singular problem one gets in the limit $\varepsilon \rightarrow 0$. At first glance, it is likely to be quite unstable because of the ambiguous term emanating from the right-hand side. Indeed, $w^{\varepsilon}$ and $z^{\varepsilon}$ suffer discontinuities at least on the lines $t=n \Delta t, n \in \mathbb{N}_{*}$, and their product with a Dirac mass has to be defined in a careful way. Anyway, we can give a precise meaning to this kind of terms in the framework proposed in [32, 36] by investigating the limit of the sequence $G\left(w^{\varepsilon}, z^{\varepsilon}\right) \partial_{t} b^{\varepsilon}$ in the weak- $\star$ topology of measures on $\mathbb{R} \times \mathbb{R}^{+}$.

The next result lies at the heart of the matter.

Proposition 1. Under the assumptions of Lemma 1, there holds:

$$
G\left(w^{\varepsilon}, z^{\varepsilon}\right) \partial_{t} b^{\varepsilon \text { weak- }-\star \mathcal{M}} \sum_{n \in \mathbb{N}_{*}} \Delta t\left(\int_{0}^{1} G(\bar{w}, \bar{z})(x, \tau) B^{\prime}(\tau) . d \tau\right) \delta(t-n \Delta t),
$$

where $\bar{w}, \bar{z}$ satisfy the following differential condition for $\tau \in[0,1]$ :

$$
\partial_{\tau}\left(\begin{array}{c}
\bar{w} \\
\bar{z}
\end{array}\right)=\mu \Delta t\left(\begin{array}{c}
G(\bar{w}, \bar{z}) \\
-G(\bar{w}, \bar{z})
\end{array}\right) B^{\prime}(\tau)
$$

completed by the initial data for $x \in \mathbb{R}$ and $t=n \Delta t, n \in \mathbb{N}_{*}$ :

$$
\bar{w}(x, 0)=w(x, t-0) ; \bar{z}(x, 0)=z(x, t-0) .
$$

The proof is actually a consequence of the following stabilization result.

Lemma 2. Assume the initial data for (44) $\left(w_{0}, z_{0}\right)$ lie in $L^{1} \cap B V(\mathbb{R})$ and that the subcharacteristic condition $c \geq\left\|f^{\prime}\left(u^{\varepsilon}\right)\right\|_{L^{\infty}}$ holds; then, the sequence $\left(w^{\nu}, z^{\nu}\right)$ of solutions of the following relaxing system

$$
\left\{\begin{array}{l}
\partial_{t} w^{\nu}+\nu c \partial_{x} w^{\nu}=G\left(w^{\nu}, z^{\nu}\right) \\
\partial_{t} z^{\nu}-\nu c \partial_{x} z^{\nu}=-G\left(w^{\nu}, z^{\nu}\right)
\end{array}\right.
$$

is relatively compact in $L_{\text {loc }}^{1}(\mathbb{R} \times] 0, T[)$ as $\nu \rightarrow 0$.

Proof. It follows the lines of the one given for Lemma 1

- Multiplying (11) by $\left(\operatorname{sgn}\left(w^{\nu}\right), \operatorname{sgn}\left(z^{\nu}\right)\right)$ and integrating over $x \in \mathbb{R}$ leads to

$$
\partial_{t} \int_{\mathbb{R}}\left(\left|w^{\nu}\right|+\left|z^{\nu}\right|\right)(x, t) \cdot d x \leq 0,
$$

after having used both the mean-value theorem and the subcharacteristic condition.

- Differentiating (II) with respect to $x$, multiplying by $\left(\operatorname{sgn}\left(\partial_{x} w^{\nu}\right), \operatorname{sgn}\left(\partial_{x} z^{\nu}\right)\right)$ and integrating over $x \in \mathbb{R}$ gives:

$$
\partial_{t} \int_{\mathbb{R}}\left(\left|\partial_{x} w^{\nu}\right|+\left|\partial_{x} z^{\nu}\right|\right)(x, t) \cdot d x \leq 0 .
$$

- Integrating (11) with respect to time gives the $L^{1}$-equicontinuity property for $t>t_{0}$ :

$$
\begin{aligned}
& \int_{\mathbb{R}}\left|w^{\nu}(x, t)-w^{\nu}\left(x, t_{0}\right)\right|+\left|z^{\nu}(x, t)-z^{\nu}\left(x, t_{0}\right)\right| \cdot d x \\
& \quad \leq\left|t-t_{0}\right|\left[\nu c\left(T V\left(w_{0}\right)+T V\left(z_{0}\right)\right)+2\left(\left\|w_{0}\right\|_{L^{1}(\mathbb{R})}+\left\|z_{0}\right\|_{L^{1}(\mathbb{R})}\right)\right] .
\end{aligned}
$$

This completes the proof of Lemma 2 
We can now state the proof of Proposition 1 .

Proof. First of all, $G\left(w^{\varepsilon}, z^{\varepsilon}\right)$ is uniformly bounded and relatively compact in the strong $L_{\text {loc }}^{1}$-topology and $\partial_{t} b^{\varepsilon}$ converges in the weak- $\star$ topology of measures. Their product is therefore weakly convergent as a measure. Let $\phi$ be a continuous function with compact support in $\mathbb{R} \times \mathbb{R}_{*}^{+}$; according to $(7)$, the object under the scope is:

$$
\begin{aligned}
\int_{\mathbb{R} \times \mathbb{R}_{*}^{+}} G\left(w^{\varepsilon}, z^{\varepsilon}\right)(x, t) \partial_{t} b^{\varepsilon}(t) \phi(x, t) d x . d t \\
=\sum_{n \in \mathbb{N}_{*}} \int_{\mathbb{R}} \int_{n \Delta t-\varepsilon}^{n \Delta t} G\left(w^{\varepsilon}, z^{\varepsilon}\right)(x, t) \frac{\Delta t}{\varepsilon} B^{\prime}\left(1+\frac{t-n \Delta t}{\varepsilon}\right) \phi(x, t) . d x . d t .
\end{aligned}
$$

We pick an integer $n \in \mathbb{N}_{*}$ and we just perform a change to the "inner variable" $\tau$ by introducing $\tau=1+\frac{t-n \Delta t}{\varepsilon} \in[0,1]$. Therefore, the "inner system" holds for $\varepsilon>0$ :

$$
\left\{\begin{array}{l}
\partial_{\tau} w^{\varepsilon}+\varepsilon c \partial_{x} w^{\varepsilon}=\mu G\left(w^{\varepsilon}, z^{\varepsilon}\right) \Delta t \cdot B^{\prime}(\tau), \\
\partial_{\tau} z^{\varepsilon}-\varepsilon c \partial_{x} z^{\varepsilon}=-\mu G\left(w^{\varepsilon}, z^{\varepsilon}\right) \Delta t \cdot B^{\prime}(\tau),
\end{array}\right.
$$

together with the initial data:

$$
w^{\varepsilon}(x, \tau=0)=w^{\varepsilon}(x, n \Delta t-\varepsilon) ; z^{\varepsilon}(x, \tau=0)=z^{\varepsilon}(x, n \Delta t-\varepsilon) .
$$

Lemma 2 implies that $w^{\varepsilon}, z^{\varepsilon}$ satisfy (9) in the limit $\varepsilon \rightarrow 0$. Therefore, we get:

$$
\begin{aligned}
\int_{\mathbb{R} \times \mathbb{R}_{*}^{+}} G\left(w^{\varepsilon}, z^{\varepsilon}\right)(x, t) \partial_{t} b^{\varepsilon}(t) \phi(x, t) \cdot d x \cdot d t \\
=\sum_{n \in \mathbb{N}_{*}} \int_{\mathbb{R}} \int_{0}^{1} G\left(w^{\varepsilon}, z^{\varepsilon}\right)(x, \tau) \frac{\Delta t}{\varepsilon} B^{\prime}(\tau) \phi(x, \varepsilon \tau-\varepsilon+n \Delta t) . d x . \varepsilon d \tau .
\end{aligned}
$$

Sending $\varepsilon$ to zero in the previous formula completes the proof of Proposition 1

2.4. From a contraction property to the Riemann problem. The meaning we found for the instable product gives us in return the following useful property.

Lemma 3. Under the subcharacteristic condition and for every initial data $\left(w_{0}, z_{0}\right)$ belonging to $L^{1} \cap B V(\mathbb{R})$, the following singular relaxing system:

$$
\left\{\begin{array}{l}
\partial_{t} w+c \partial_{x} w=\mu \sum_{n \in \mathbb{N}_{*}} \Delta t . G(w, z) \delta(t-n \Delta t), \\
\partial_{t} z-c \partial_{x} z=-\mu \sum_{n \in \mathbb{N}_{*}} \Delta t . G(w, z) \delta(t-n \Delta t)
\end{array}\right.
$$

is $L^{1}(\mathbb{R})$-contractive.

Of course, here and hereafter, the right-hand side of (12) is to be understood by means of (8), (91), (10) as stated in Proposition 1

Proof. Consider two sets of initial data $\left(w_{0}^{1}, z_{0}^{1}\right)$ and $\left(w_{0}^{2}, z_{0}^{2}\right)$ for (12). We subtract the two equations on $w$, we multiply by $\operatorname{sgn}\left(w^{1}-w^{2}\right)$ and we integrate over $x \in \mathbb{R}$. We do the same for the equations on $z$. By linearity of the convective parts, the space terms drop; it just remains to study the two nonlinear right-hand sides. We 
have:

$$
\begin{aligned}
\partial_{t} \int_{\mathbb{R}}\left|w^{1}-w^{2}\right|(x, t) \cdot d x & =\mu \sum_{n \in \mathbb{N}_{*}} \Delta t \cdot \operatorname{sgn}\left(w^{1}-w^{2}\right)(x, t) \\
& \times\left\{\int_{0}^{1}\left[G\left(\bar{w}^{1}, \bar{z}^{1}\right)-G\left(\bar{w}^{2}, \bar{z}^{2}\right)\right](x, \tau) B^{\prime}(\tau) \cdot d \tau\right\} \delta(t-n \Delta t)
\end{aligned}
$$

together with:

$$
\begin{aligned}
\partial_{t} \int_{\mathbb{R}}\left|z^{1}-z^{2}\right|(x, t) \cdot d x & =-\mu \sum_{n \in \mathbb{N}_{*}} \Delta t \cdot \operatorname{sgn}\left(z^{1}-z^{2}\right)(x, t) \\
\times & \left\{\int_{0}^{1}\left[G\left(\bar{w}^{1}, \bar{z}^{1}\right)-G\left(\bar{w}^{2}, \bar{z}^{2}\right)\right](x, \tau) B^{\prime}(\tau) \cdot d \tau\right\} \delta(t-n \Delta t) .
\end{aligned}
$$

On the one hand, we can use the mean-value theorem to linearize the jump on $G$ :

$$
G\left(\bar{w}^{1}, \bar{z}^{1}\right)-G\left(\bar{w}^{2}, \bar{z}^{2}\right)=\left\langle\nabla G(\xi),\left(\begin{array}{c}
\bar{w}^{1}-\bar{w}^{2} \\
\bar{z}^{1}-\bar{z}^{2}
\end{array}\right)\right\rangle_{\mathbb{R}^{2}}
$$

and on the other hand, we have that the flow of the differential system (9) is orderpreserving which means that for $\tau \in] 0,1]$ :

$$
\begin{aligned}
\operatorname{sgn}\left(\bar{w}^{1}-\bar{w}^{2}\right)(x, \tau) & =\operatorname{sgn}\left(\bar{w}^{1}-\bar{w}^{2}\right)(x, t-0), \\
\operatorname{sgn}\left(\bar{z}^{1}-\bar{z}^{2}\right)(x, \tau) & =\operatorname{sgn}\left(\bar{z}^{1}-\bar{z}^{2}\right)(x, t-0) .
\end{aligned}
$$

Since the sign-functions are constant in $\tau$, we can throw them inside the integrals in (13), (14) and sum both the resulting equations. Therefore, these right-hand sides cancel and we end up with:

$$
\partial_{t} \int_{\mathbb{R}}\left(\left|w^{1}-w^{2}\right|+\left|z^{1}-z^{2}\right|\right)(x, t) \cdot d x \leq 0 .
$$

This completes the proof.

Actually, Proposition 1 also provides us with the way to uniquely solve the Riemann problem for the singular system (12) in $\mathbb{R} \times[0, \Delta t]$. That reduces to selecting the following piecewise constant initial data:

$$
\begin{aligned}
& w_{0}(x)=\left\{\begin{array}{lll}
w_{L} & \text { if } & x<0, \\
w_{R} & \text { if } & x>0
\end{array}\right. \\
& z_{0}(x)=\left\{\begin{array}{lll}
z_{L} & \text { if } & x<0 \\
z_{R} & \text { if } & x>0
\end{array}\right.
\end{aligned}
$$

Thanks to the linear-free convective part, we have for $t<\Delta t$ :

$$
w(x, t)=w_{0}(x-c t) ; z(x, t)=z_{0}(x+c t) .
$$

The time $t=\Delta t$ corresponds to the projection stage onto the local Maxwellian. At this level, it is useful to recall a result from [1]:

Lemma 4. Assume that $B^{\prime} \equiv 1$; the solution of (91), (10) reads:

$$
\left\{\begin{array}{l}
\bar{w}(x, \tau)=\bar{w}(x, 0)+(1-\exp (-\mu \Delta t \tau)) G(\bar{w}, \bar{z})(x, 0) \\
\bar{z}(x, \tau)=\bar{z}(x, 0)-(1-\exp (-\mu \Delta t \tau)) G(\bar{w}, \bar{z})(x, 0)
\end{array}\right.
$$


Then, as an immediate consequence of Lemma 4, we can write down the solution of (12), (15):

$$
\begin{aligned}
& w(x, \Delta t)=\left\{\begin{array}{lll}
w_{L}+(1-\exp (-\mu \Delta t)) G\left(w_{L}, z_{L}\right) & \text { if } & x<-c \Delta t, \\
w_{L}+(1-\exp (-\mu \Delta t)) G\left(w_{L}, z_{R}\right) & \text { if } & -c \Delta t<x<c \Delta t, \\
w_{R}+(1-\exp (-\mu \Delta t)) G\left(w_{R}, z_{R}\right) & \text { if } & x>c \Delta t,
\end{array}\right. \\
& z(x, \Delta t)=\left\{\begin{array}{lll}
z_{L}-(1-\exp (-\mu \Delta t)) G\left(w_{L}, z_{L}\right) & \text { if } & x<-c \Delta t, \\
z_{R}-(1-\exp (-\mu \Delta t)) G\left(w_{L}, z_{R}\right) & \text { if } & -c \Delta t<x<c \Delta t, \\
z_{R}-(1-\exp (-\mu \Delta t)) G\left(w_{R}, z_{R}\right) & \text { if } & x>c \Delta t .
\end{array}\right.
\end{aligned}
$$

2.5. Convergence of the relaxing scheme. For simplicity only, we suppose from now on that $B^{\prime} \equiv 1$ in order to be able to use the explicit formulas (17). The next step is to study the properties of a relaxing scheme in the sense of [28, 29] based on local Riemann problems of the type (12), (15), (18). More precisely, given any initial data $\left(w_{0}, z_{0}\right)$ in $L^{1} \cap B V(\mathbb{R})$ and a space-step $h>0$, we define the following sequences for $j \in \mathbb{Z}$ :

$$
w_{j}^{0}=\frac{1}{h} \int_{\left(j-\frac{1}{2}\right) h}^{\left(j+\frac{1}{2}\right) h} w_{0}(x) \cdot d x ; z_{j}^{0}=\frac{1}{h} \int_{\left(j-\frac{1}{2}\right) h}^{\left(j+\frac{1}{2}\right) h} z_{0}(x) \cdot d x .
$$

Then we define the piecewise constant approximations $\left(w^{h}, z^{h}\right)$ the following way:

$$
\left.\begin{array}{c}
w^{h}(x, t)=w_{j}^{n} \\
z^{h}(x, t)=z_{j}^{n}
\end{array}\right\} \text { for }(x, t) \in\left[\left(j-\frac{1}{2}\right) h,\left(j+\frac{1}{2}\right) h[\times[n \Delta t,(n+1) \Delta t[.\right.
$$

Now, we must state how we propose to update the values $w_{j}^{n}, z_{j}^{n}$ as times increase: one possible way is to exploit the simple structure of the singular Riemann problems previously studied in order to define a relaxing upwind scheme. More precisely, under the restriction $\frac{c \Delta t}{h} \leq \frac{1}{2}$, the Godunov scheme for (12) reads:

$$
\begin{aligned}
w_{j}^{n+1}= & w_{j}^{n}-\frac{c \Delta t}{h}\left(w_{j}^{n}-w_{j-1}^{n}\right)+(1-\exp (-\mu \Delta t)) G\left(w_{j}^{n}, z_{j}^{n}\right) \\
& +\frac{c \Delta t}{h}(1-\exp (-\mu \Delta t))\left(G\left(w_{j-1}^{n}, z_{j}^{n}\right)-2 G\left(w_{j}^{n}, z_{j}^{n}\right)+G\left(w_{j}^{n}, z_{j+1}^{n}\right)\right), \\
z_{j}^{n+1}= & z_{j}^{n}+\frac{c \Delta t}{h}\left(z_{j+1}^{n}-z_{j}^{n}\right)-(1-\exp (-\mu \Delta t)) G\left(w_{j}^{n}, z_{j}^{n}\right) \\
& -\frac{c \Delta t}{h}(1-\exp (-\mu \Delta t))\left(G\left(w_{j-1}^{n}, z_{j}^{n}\right)-2 G\left(w_{j}^{n}, z_{j}^{n}\right)+G\left(w_{j}^{n}, z_{j+1}^{n}\right)\right) .
\end{aligned}
$$

At this level, we point out that this approach is quite close to the one which has been studied by F. Bouchut, [4] and A. Vasseur, 40], in the context of kinetic equations. It is also related to the modified split-scheme proposed in [25] in the context of reactive Euler equations. At last, the split-schemes investigated in [1] are obtained just by inserting a projection stage onto piecewise constant functions similar to (19) between the free transport step and the relaxation process, that is, right between (16) and (18).

The next lemma establishes the $L^{1}$-stability for this approach. 
Lemma 5. Assume the CFL restriction $\frac{c \Delta t}{h} \leq \frac{1}{2}$ and the subcharacteristic condition $c \geq\left\|f^{\prime}\left(u^{h}\right)\right\|_{L^{\infty}}$. Then, as $\left(w_{0}, z_{0}\right) \in L^{1} \cap B V(\mathbb{R})$, the following estimates hold:

$$
\begin{aligned}
\left\|w^{h}(., n \Delta t)\right\|_{L^{1}(\mathbb{R})}+\left\|z^{h}(., n \Delta t)\right\|_{L^{1}(\mathbb{R})} & \leq\left\|w_{0}\right\|_{L^{1}(\mathbb{R})}+\left\|z_{0}\right\|_{L^{1}(\mathbb{R})}, \\
\left\|w^{h}(., n \Delta t)\right\|_{L^{\infty}(\mathbb{R})}+\left\|z^{h}(., n \Delta t)\right\|_{L^{\infty}(\mathbb{R})} & \leq\left\|w_{0}\right\|_{L^{\infty}(\mathbb{R})}+\left\|z_{0}\right\|_{L^{\infty}(\mathbb{R})}, \\
T V\left(w^{h}(., n \Delta t)\right)+T V\left(z^{h}(., n \Delta t)\right) & \leq T V\left(w_{0}\right)+T V\left(z_{0}\right) .
\end{aligned}
$$

Proof. We are still using the arguments presented in the proof of Lemma 1 Of course, things are more tedious at this numerical level because of the indices.

- $L^{1}$ - and $L^{\infty}$-bounds; we use the mean-value theorem and the fact that $G(0,0)=0$ to derive thanks to the special form of $G,(5)$ :

$$
G\left(w_{j-1}^{n}, z_{j}^{n}\right)=\partial_{w} G\left(\xi_{j-1}^{n}, z_{j}^{n}\right) w_{j-1}^{n}+\partial_{z} G\left(w_{j-1}^{n}, \zeta_{j}^{n}\right) z_{j}^{n} .
$$

It follows that the scheme (21) on $w$ becomes:

$$
\begin{aligned}
w_{j}^{n+1}= & \left(1-\frac{c \Delta t}{h}\right) w_{j}^{n}+\frac{c \Delta t}{h} w_{j-1}^{n} \\
& +(1-\exp (-\mu \Delta t))\left(1-2 \frac{c \Delta t}{h}\right)\left(\partial_{w} G\left(\xi_{j}^{n}, z_{j}^{n}\right) w_{j}^{n}+\partial_{z} G\left(w_{j}^{n}, \zeta_{j}^{n}\right) z_{j}^{n}\right) \\
& +(1-\exp (-\mu \Delta t)) \frac{c \Delta t}{h}\left(\partial_{w} G\left(\xi_{j-1}^{n}, z_{j}^{n}\right) w_{j-1}^{n}+\partial_{z} G\left(w_{j-1}^{n}, \zeta_{j}^{n}\right) z_{j}^{n}\right) \\
& +(1-\exp (-\mu \Delta t)) \frac{c \Delta t}{h}\left(\partial_{w} G\left(\xi_{j}^{n}, z_{j+1}^{n}\right) w_{j}^{n}+\partial_{z} G\left(w_{j}^{n}, \zeta_{j+1}^{n}\right) z_{j+1}^{n}\right) .
\end{aligned}
$$

The one on $z$ is similar. Under the subcharacteristic condition, we have $\partial_{w} G \leq 0$ and $\partial_{z} G \geq 0$. Let us now assume that we are allowed to take the moduli in the previous expressions which are to be checked later. Therefore, $\left|w_{j}^{n+1}\right|$ can be bounded by a convex combination of the values $\left|w_{j-1}^{n}\right|,\left|w_{j}^{n}\right|$, $\left|z_{j}^{n}\right|,\left|z_{j+1}^{n}\right|:$

$$
\begin{aligned}
\left|w_{j}^{n+1}\right| \leq \mid & \left|w_{j}^{n}\right|\left[\left(1-\frac{c \Delta t}{h}\right)+(1-\exp (-\mu \Delta t))\right. \\
& \left.\times\left(\left(1-2 \frac{c \Delta t}{h}\right) \partial_{w} G\left(\xi_{j}^{n}, z_{j}^{n}\right)+\frac{c \Delta t}{h} \partial_{w} G\left(\xi_{j}^{n}, z_{j+1}^{n}\right)\right)\right] \\
+ & \left|w_{j-1}^{n}\right| \frac{c \Delta t}{h}\left[1+(1-\exp (-\mu \Delta t)) \partial_{w} G\left(\xi_{j-1}^{n}, z_{j}^{n}\right)\right] \\
+ & \left|z_{j}^{n}\right|(1-\exp (-\mu \Delta t)) \\
& \times\left[\frac{c \Delta t}{h} \partial_{z} G\left(w_{j-1}^{n}, \zeta_{j}^{n}\right)+\left(1-2 \frac{c \Delta t}{h}\right) \partial_{z} G\left(w_{j}^{n}, \zeta_{j}^{n}\right)\right] \\
+ & \left|z_{j+1}^{n}\right|(1-\exp (-\mu \Delta t)) \frac{c \Delta t}{h} \partial_{z} G\left(w_{j}^{n}, \zeta_{j+1}^{n}\right) .
\end{aligned}
$$

We proceed the same way for $\left|z_{j}^{n+1}\right|$ which is bounded by a convex combination of $\left|z_{j}^{n}\right|,\left|z_{j+1}^{n}\right|,\left|w_{j-1}^{n}\right|,\left|w_{j}^{n}\right|$. Adding these two expressions leads to:

$$
\left|w_{j}^{n+1}\right|+\left|z_{j}^{n+1}\right| \leq\left(1-\frac{c \Delta t}{h}\right)\left(\left|w_{j}^{n}\right|+\left|z_{j}^{n}\right|\right)+\frac{c \Delta t}{h}\left|w_{j-1}^{n}\right|+\frac{c \Delta t}{h}\left|z_{j+1}^{n}\right| .
$$

This clearly implies the first two announced bounds. 
- BV-bound; now we use another kind of linearization of the nonlinear term. Namely, we rewrite $w_{j}^{n+1}$ the following way:

$$
\begin{aligned}
w_{j}^{n+1}=w_{j}^{n} & -\frac{c \Delta t}{h}\left(w_{j}^{n}-w_{j-1}^{n}\right)+(1-\exp (-\mu \Delta t)) G\left(w_{j}^{n}, z_{j}^{n}\right) \\
& -\frac{c \Delta t}{h}(1-\exp (-\mu \Delta t)) \partial_{w} G\left(\xi_{j-\frac{1}{2}}^{n}, z_{j}^{n}\right)\left(w_{j}^{n}-w_{j-1}^{n}\right) \\
& +\frac{c \Delta t}{h}(1-\exp (-\mu \Delta t)) \partial_{w} G\left(w_{j}^{n}, \zeta_{j+\frac{1}{2}}^{n}\right)\left(z_{j+1}^{n}-z_{j}^{n}\right) .
\end{aligned}
$$

We write down the expressions for $w_{j}^{n+1}-w_{j-1}^{n+1}$ and $z_{j}^{n+1}-z_{j-1}^{n+1}$. Once again, we suppose $\Delta t$ and $h$ are adjusted in such a way that all the incremental coefficients are positive and we take the moduli. We end up with:

$$
\begin{aligned}
\mid w_{j}^{n+1} & -w_{j-1}^{n+1} \mid \\
\leq & \left|w_{j}^{n}-w_{j-1}^{n}\right|\left(1-\frac{c \Delta t}{h}\right)\left(1+(1-\exp (-\mu \Delta t)) \partial_{w} G\left(\xi_{j-\frac{1}{2}}^{n}, z_{j}^{n}\right)\right) \\
& +\left|w_{j-1}^{n}-w_{j-2}^{n}\right| \frac{c \Delta t}{h}\left(1+(1-\exp (-\mu \Delta t)) \partial_{w} G\left(\xi_{j-\frac{3}{2}}^{n}, z_{j-1}^{n}\right)\right) \\
& +\left|z_{j+1}^{n}-z_{j}^{n}\right| \frac{c \Delta t}{h}(1-\exp (-\mu \Delta t)) \partial_{z} G\left(w_{j}^{n}, \zeta_{j+\frac{1}{2}}^{n}\right) \\
& +\left|z_{j}^{n}-z_{j-1}^{n}\right|\left(1-\frac{c \Delta t}{h}\right)(1-\exp (-\mu \Delta t)) \partial_{z} G\left(w_{j-1}^{n}, \zeta_{j-\frac{1}{2}}^{n}\right),
\end{aligned}
$$

together with a similar bound for $\left|z_{j}^{n+1}-z_{j-1}^{n+1}\right|$. We add the two inequalities and after the cancellations, we get:

$$
\begin{aligned}
T V\left(w^{n+1}\right) \leq & \sum_{j \in \mathbb{Z}}\left|w_{j}^{n}-w_{j-1}^{n}\right|\left(1+(1-\exp (-\mu \Delta t)) \partial_{w} G\left(\xi_{j-\frac{1}{2}}^{n}, z_{j}^{n}\right)\right) \\
& +\sum_{j \in \mathbb{Z}}\left|z_{j}^{n}-z_{j-1}^{n}\right|(1-\exp (-\mu \Delta t)) \partial_{z} G\left(w_{j-1}^{n}, \zeta_{j-\frac{1}{2}}^{n}\right),
\end{aligned}
$$

together with:

$$
\begin{aligned}
T V\left(z^{n+1}\right) \leq & \sum_{j \in \mathbb{Z}}\left|z_{j}^{n}-z_{j-1}^{n}\right|\left(1-(1-\exp (-\mu \Delta t)) \partial_{z} G\left(w_{j-1}^{n}, \zeta_{j-\frac{1}{2}}^{n}\right)\right) \\
& +\sum_{j \in \mathbb{Z}}\left|w_{j}^{n}-w_{j-1}^{n}\right|(1-\exp (-\mu \Delta t)) \partial_{w} G\left(\xi_{j-\frac{1}{2}}^{n}, z_{j}^{n}\right) .
\end{aligned}
$$

It remains to add both inequalities to derive:

$$
T V\left(w^{n+1}\right)+T V\left(z^{n+1}\right) \leq T V\left(w_{0}\right)+T V\left(z_{0}\right) .
$$

At this level, we are done provided we can prove that all the incremental coefficients on which the $L^{1 / \infty}$ and the BV bounds rely are in fact positive. We study therefore:

$$
\left\{\begin{array}{l}
\alpha=1+(1-\exp (-\mu \Delta t)) \partial_{w} G(w, z) \\
\beta=1-(1-\exp (-\mu \Delta t)) \partial_{z} G(w, z) .
\end{array}\right.
$$

Since we know that

$$
\left(\frac{1-\exp (-\mu \Delta t)}{1+\exp (-\mu \Delta t)}\right) \in[0,1]
$$


the positivity of $\alpha$ and $\beta$ is a direct consequence of the subcharacteristic condition since $c$ dominates $\left\|f^{\prime}\left(u^{h}\right)\right\|_{L^{\infty}}$. This is enough for the BV-bound. Now, for the $L^{1 / \infty}$ ones, we also need to check the following quantities:

$$
\begin{aligned}
& \alpha^{\prime}=\left(1-\frac{c \Delta t}{h}\right)+(1-\exp (-\mu \Delta t))\left[\left(1-2 \frac{c \Delta t}{h}\right) \partial_{w} G(w, z)+\frac{c \Delta t}{h} \partial_{w} G\left(w, z^{\prime}\right)\right], \\
& \beta^{\prime}=\left(1-\frac{c \Delta t}{h}\right)-(1-\exp (-\mu \Delta t))\left[\left(1-2 \frac{c \Delta t}{h}\right) \partial_{z} G(w, z)+\frac{c \Delta t}{h} \partial_{z} G\left(w^{\prime}, z\right)\right] .
\end{aligned}
$$

This is a consequence of the CFL restriction $\frac{c \Delta t}{h} \leq \frac{1}{2}$ since under this assumption, both $\alpha^{\prime}$ and $\beta^{\prime}$ are bigger than:

$$
\left(1-\frac{c \Delta t}{h}\right) \exp (-\mu \Delta t) \geq 0 \text {. }
$$

This completes the proof of Lemma 5

Lemma 6. Under the assumptions of Lemma 5, the relaxation estimate holds:

$$
\begin{aligned}
\left\|G\left(w^{h}, z^{h}\right)(., n \Delta t)\right\|_{L^{1}(\mathbb{R}) \leq} \leq & \exp (-\mu \Delta t)\left\|G\left(w^{h}, z^{h}\right)(.,(n-1) \Delta t)\right\|_{L^{1}(\mathbb{R})} \\
& +O(h)\left[T V\left(w^{0}\right)+T V\left(z^{0}\right)\right] .
\end{aligned}
$$

Moreover, $w^{h}$ and $z^{h}$ are $L^{1}$-equicontinuous with respect to the $t$ variable.

Proof. - Relaxation estimate; we just use the mean-value theorem to write that:

$$
\begin{aligned}
G\left(w_{j}^{n+1}, z_{j}^{n+1}\right)= & \frac{1}{2}\left(-w_{j}^{n+1}+z_{j}^{n+1}\right)-f\left(-\frac{w_{j}^{n+1}+z_{j}^{n+1}}{2 c}\right) \\
= & G\left(w_{j}^{n}, z_{j}^{n}\right) \\
& -\frac{1}{2 c}\left[\left(c-f^{\prime}(\kappa)\right)\left(w_{j}^{n+1}-w_{j}^{n}\right)-\left(c+f^{\prime}(\kappa)\right)\left(z_{j}^{n+1}-z_{j}^{n}\right)\right] .
\end{aligned}
$$

Inserting (23) and its analogue for $z_{j}^{n+1}-z_{j}^{n}$ gives the announced estimate after taking the moduli and summing over $j \in \mathbb{Z}$.

- $L^{1}$-equicontinuity in time; it is sufficient to multiply (23) by $h$ and to derive a similar relation for $h\left(z_{j}^{n+1}-z_{j}^{n}\right)$. Therefore, we take the moduli and sum over $j \in \mathbb{Z}$ to get:

$$
\begin{aligned}
& \left\|w^{h}(.,(n+1) \Delta t)-w^{h}(., n \Delta t)\right\|_{L^{1}(\mathbb{R})}+\left\|z^{h}(.,(n+1) \Delta t)-z^{h}(., n \Delta t)\right\|_{L^{1}(\mathbb{R})} \\
& \quad \leq O(\Delta t)\left[T V\left(w_{0}\right)+T V\left(z_{0}\right)+\left(\frac{1-\exp (-\mu \Delta t)}{\Delta t}\right)\left\|G\left(w^{h}, z^{h}\right)(., n \Delta t)\right\|_{L^{1}(\mathbb{R})}\right] .
\end{aligned}
$$

We have in particular that $\left(w^{h}, z^{h}\right) \in L^{1} \cap B V(\mathbb{R} \times[0, T])$ uniformly in $\mu$ for $h>0$. These estimates are meaningful since we have:

$$
\forall n \in \mathbb{N}, T V\left(u^{h}(., n \Delta t)\right) \leq \frac{1}{2 c}\left(T V\left(w^{h}(., n \Delta t)\right)+T V\left(z^{h}(., n \Delta t)\right)\right) .
$$

The estimate (24) suggests that the distance between the approximate solutions $\left(w^{h}, z^{h}\right)$ and the equilibrium manifold is a bit greater for $\mu$ finite and $h>0$ (the situation encountered in practice) in the case of our scheme (21) compared to a classical splitting algorithm (see for instance [1]) for which one gets

$$
\begin{aligned}
\exp (\mu \Delta t)\left\|G\left(w^{h}, z^{h}\right)(., n \Delta t)\right\|_{L^{1}(\mathbb{R}) \leq} & \left\|G\left(w^{h}, z^{h}\right)(.,(n-1) \Delta t)\right\|_{L^{1}(\mathbb{R})} \\
& +O(\Delta t)\left[T V\left(w^{0}\right)+T V\left(z^{0}\right)\right]
\end{aligned}
$$


instead of (24). This will be checked numerically later on. Anyway, it is sufficient to conclude.

Theorem 1. Assume that $v_{0}=f\left(u_{0}\right)$, the CFL restriction $\frac{c \Delta t}{h} \leq \frac{1}{2}$ and the subcharacteristic condition $c \geq\left\|f^{\prime}\left(u^{h}\right)\right\|_{L^{\infty}}$; then, as $\mu \Delta t \rightarrow+\infty, h \rightarrow 0$, the sequence of numerical approximations $u^{h}$ generated by (21) converges strongly in $L_{\text {loc }}^{1}(\mathbb{R} \times] 0, T[)$ towards $u$, the entropy solution in the sense of Kružkov to:

$$
\left\{\begin{array}{l}
\partial_{t} u+\partial_{x} f(u)=0 \\
u(., 0)=u_{0} \in L^{1} \cap B V(\mathbb{R}) .
\end{array}\right.
$$

Proof. Of course, we use the fact that $u^{h}=\frac{-1}{2 c}\left(w^{h}+z^{h}\right)$. We add the two equalities in (21) to get:

$$
u_{j}^{n+1}=u_{j}^{n}-\frac{\Delta t}{2 h}\left(\left(z_{j+1}^{n}-w_{j}^{n}\right)-\left(z_{j}^{n}-w_{j-1}^{n}\right)\right) .
$$

Thanks to Lemmas 5 and 6 and in particular to the relaxation estimate (24), we can write the associated relaxed scheme:

$$
u_{j}^{n+1}=u_{j}^{n}-\frac{\Delta t}{2 h}\left(f\left(u_{j+1}^{n}\right)-f\left(u_{j-1}^{n}\right)\right)+\frac{c \Delta t}{2 h}\left(u_{j+1}^{n}-2 u_{j}^{n}+u_{j-1}^{n}\right)+O(h) .
$$

This Lax-Friedrichs type scheme inherits all the preceding bounds under the restrictions prescribed in Theorem 1. Therefore, we can pass to the limits $\mu \Delta t \rightarrow+\infty$, $h \rightarrow 0$. The entropy condition is enforced by the monotonicity property of this relaxed scheme and we can apply the results of [10].

2.6. Numerical results. We performed some computational experiments on the following test case with these two initial data:

$$
\begin{gathered}
\partial_{t} u+\partial_{x}\left(\frac{u^{2}}{2}\right)=0 \text { with } x \in \mathbb{R} \text { and } 0<t \leq 0.3, \\
u_{0}(x)=\left\{\begin{array}{ll}
1 & \text { if } \quad x<0, \\
0 & \text { if } \quad x>0,
\end{array} \text { and } u_{0}(x)=\left\{\begin{array}{lll}
0 & \text { if } & x<0, \\
1 & \text { if } & x>0 .
\end{array}\right.\right.
\end{gathered}
$$

The entropy solution is a shock moving at speed $\sigma=\frac{1}{2}$ for the first initial datum and a rarefaction wave for the second one. We compared the results obtained with the scheme (21) and a time splitting algorithm for which the convection and the relaxation stages are handled separately. In both approaches, we used the exact solutions (17). On Figures 11 and 2, we display the decay of the sup-norm of $(v-f(u))$ as $\frac{1}{\mu} \rightarrow 0$ at time $T=0.3$ for both schemes. According to the relaxation estimate (24), we observe the expected convergence, but the size of the relaxation term stalls at a certain value for our "upwind relaxation scheme". This shows in particular that our estimate is optimal since it can be observed computationally. The time splitting technique seems therefore to be a well-suited approach for the discretization of relaxation systems to the scalar conservation law in the limit $\mu \rightarrow+\infty$. For the computation of intermediate regimes for which $\mu$ remains finite, the two approaches nearly coincide. The parameters used in these runs were: $\Delta t=0.005, h=0.015, c=2$. 


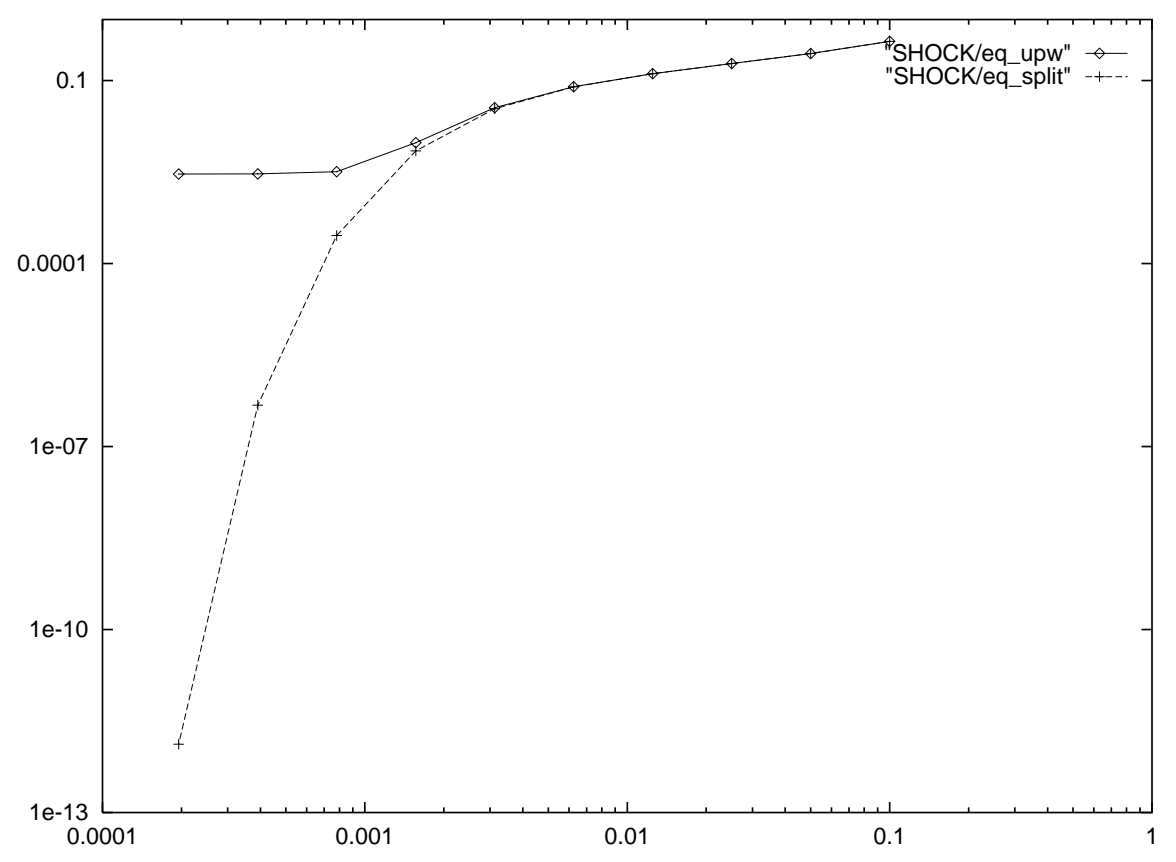

Figure 1. Sup-norm decay of the relaxation term for (25) as $\frac{1}{\mu} \rightarrow 0$ : shock wave.

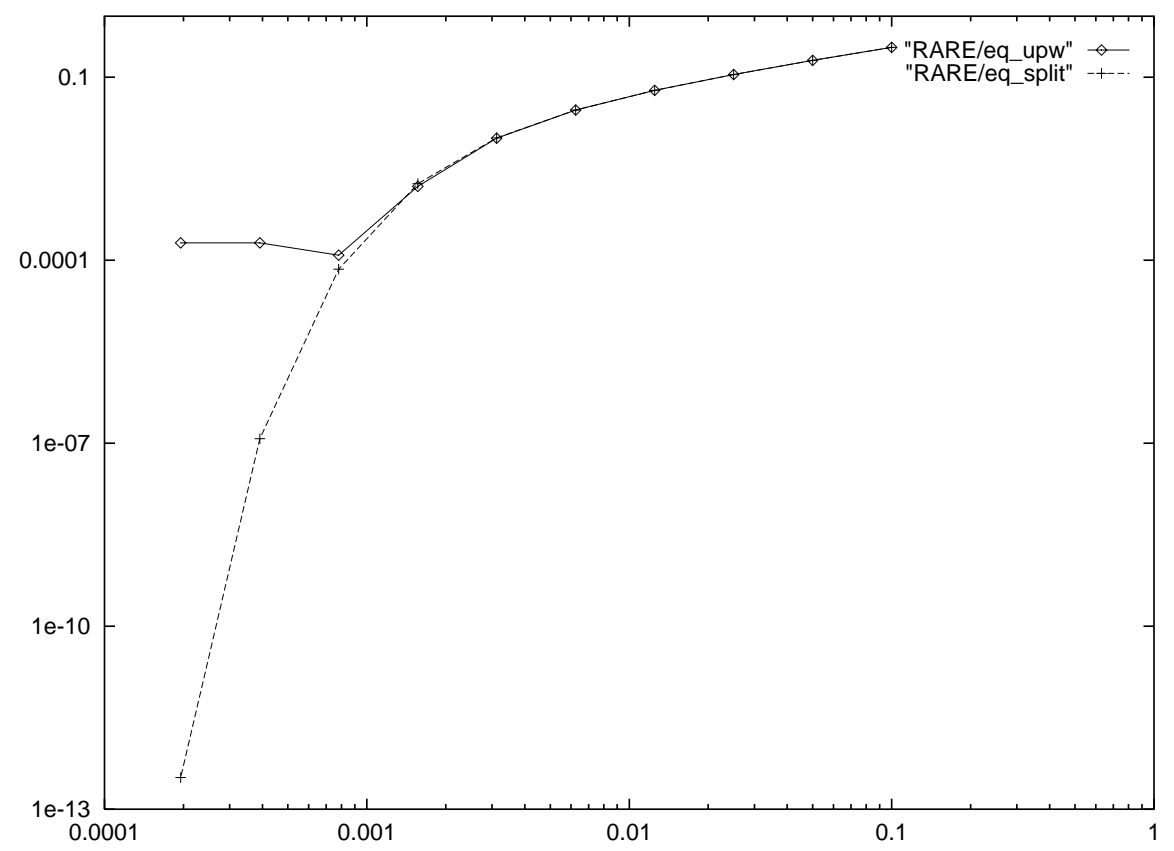

Figure 2. Sup-norm decay of the relaxation term for (25) as $\frac{1}{\mu} \rightarrow 0$ : rarefaction wave. 


\section{General Source terms in SCALAR BALANCE LAWS}

3.1. Motivation. We are now interested in the numerical approximation of the entropy solution, 31], to the general scalar balance law:

$$
\left\{\begin{array}{l}
\partial_{t} u+\partial_{x} f(u)=k(x) g(u) \\
u(., 0)=u_{0} \in B V(\mathbb{R}) .
\end{array}\right.
$$

Later on, we will make several assumptions on the functions $f, g, k$ in order to ensure that (26) is well posed as a Cauchy problem.

Numerous numerical schemes have been proposed for solving this equation (see for instance the references quoted in [19, 20]) relying on generalized Riemann problems, time splitting techniques, methods of lines ... Unfortunately, these existing approaches are not totally satisfying for at least two reasons:

- the stiff regimes corresponding to a function $k$ whose $C^{1}$ norm is big may not be properly handled: see, e.g., [18, 33] and references therein;

- the solutions one gets at numerical steady-state may be very poor approximations of the expected large time behavior of the original equation: see, e.g., [7, 8, 13, 14, 19, 20].

Recently, Greenberg and LeRoux have proposed to treat the particular case $g(u)=-u$ in a different way inside a Godunov type scheme, 23]. One motivation was to get rid of the error coming from the projection stage lying between the treatment of the convective and the reactive part. Moreover, their construction was shown to preserve nicely the steady regimes of the differential problem. An extension followed in [22] for which it was possible to prove convergence for (26) in the case where $k$ was an arbitrary big constant without any restriction on the time step $\Delta t$ except the homogeneous CFL condition, see also [17. Still, this approach was limited to scalar problems because of the particular form the Riemann solver. One extension to the case of general systems has been made using nonconservative Riemann solvers, [19, 20]. The numerical results were very good but no real theoretical justification was proposed. Our goal here is to show rigorously that the use of these special nonconservative Riemann solvers can be justified by several compactness arguments.

For the reader's convenience, we recall here the main ideas underlying this approach. Instead of solving approximately generalized Riemann problems for (26) to derive a Godunov type scheme, we decide to solve exactly modified ones. To do so, we replace $k$ by an antiderivative $K$ and the right-hand side becomes $g(u) \partial_{x} K$. Then, we replace the smooth function $K$ by its piecewise constant approximation according to the space step $h>0$ of the selected computational grid. This way, we move from the continuous to a discrete case involving building blocks which are somewhat adjusted to the mesh size. We thus obtain a measure-valued source term which can be treated directly inside the Riemann solver by means of a simple jump relation. As the space-step is refined, $h \rightarrow 0$, it is possible to prove convergence towards the entropy solution of (26) in the context of BV functions.

3.2. Some results about initial-boundary-value problems. Before entering the core of the matter, we recall some basic results from [3, 11, 35] concerning the boundary value problem for (26):

$$
\left\{\begin{array}{l}
\left.\partial_{t} u+\partial_{x} f(u)=k(x) g(u) \text { with } x \in\right]-L, L[, t>0, \\
u(., 0)=u_{0} \in B V(-L, L), \\
u(-L, t)=u_{L}(t), u(L, t)=u_{R}(t) \text { in } B V\left(\mathbb{R}^{+}\right) .
\end{array}\right.
$$


In all the sequel, $k$ is assumed to be a positive continuously differentiable function of the $x$ variable with compact support in $\mathbb{R}$.

We will make the following general hypotheses:

$$
\left\{\begin{array}{l}
f \in C^{2}(\mathbb{R}) \text { strictly convex and } \lim _{|u| \rightarrow+\infty} f(u)=+\infty \\
u f^{\prime}(u) \geq 0 \text { for } x \in \mathbb{R}
\end{array}\right.
$$

together with:

$$
\left\{\begin{array}{l}
g \in C^{1}(\mathbb{R}) \text { and } g(0)=0, g^{\prime}(0)>0, \\
\exists U \in \mathbb{R}^{+} \text {such that }|u| \geq U \Rightarrow u g(u) \leq 0 .
\end{array}\right.
$$

It has been proved in 3,35 that (27), (28), (29) have a unique entropy solution $\mathrm{BV}([-L, L] \times[0, T])$ in the following sense:

- the following inequality holds for any $l \in \mathbb{R}$ in $\mathcal{D}^{\prime}(]-L, L[\times[0, T])$

$$
\partial_{t}|u-l|+\partial_{x}\{\operatorname{sgn}(u-l)(f(u)-f(l))\} \leq \operatorname{sgn}(u-l) k(x) g(u)
$$

- together with the boundary conditions

$$
u(-L, t) \in I^{-}\left(u_{L}\right)=\left\{\begin{array}{lll}
]-\infty,-u_{L}\right] \cup\left\{u_{L}\right\} & \text { if } & u_{L} \geq 0 \\
]-\infty, 0] & \text { if } & u_{L} \leq 0
\end{array}\right.
$$

and

$$
u(L, t) \in I^{+}\left(u_{R}\right)=\left\{\begin{array}{lll}
{[0,+\infty[} & \text { if } & u_{R} \geq 0 \\
\left\{u_{R}\right\} \cup\left[-u_{R},+\infty[\right. & \text { if } & u_{R} \leq 0 .
\end{array}\right.
$$

Under the hypotheses (28), (29), the entropy solution can be expressed by means of generalized characteristics, cf [11] and also [35].

Definition 1. A generalized characteristic associated to the entropy solution of (27) is a Lipschitz curve $\xi:[0, T] \rightarrow[-L, L]$ satisfying

$$
\dot{\xi}(t) \in\left[f^{\prime}(u(\xi(t)+0, t)), f^{\prime}(u(\xi(t)-0, t))\right] .
$$

Such a curve is a genuine characteristic in the interval $] t_{0}, t_{1}[$ if $u(\xi(t)+0, t)=$ $u(\xi(t)-0, t)$ for all $t \in] t_{0}, t_{1}[$.

Fixed $(x, t) \in[-L, L] \times[0, T]$, a backward (resp. forward) characteristic curve, satisfies (30); $; \xi(t)=x$ and is defined in $[t-\epsilon, t]$ (resp. $[t, t+\epsilon]$ ) for some $\epsilon>0$. It can be proved that for a given entropy solution of (27), (28), (29), there exists at least one forward and backward characteristic at each point $x, t$.

Proposition 2 ([11]). Assume (28), (29) and let $u$ be the entropy solution of (27). From any point $x, t \in[-L, L] \times[0, T]$, a backward maximal characteristic $\xi_{+}$and a backward minimal one $\xi_{-}$start and both are genuine. For all $s$ in their definition domain, they satisfy the following differential system:

$$
\begin{cases}\dot{\xi}_{ \pm}(s)=f^{\prime}\left(v_{ \pm}(s)\right), & \dot{v}_{ \pm}(s)=k\left(\xi_{ \pm}(s)\right) g\left(v_{ \pm}(s)\right) \\ \xi_{ \pm}(t)=x, & v_{ \pm}(t)=u(x \pm 0, t)\end{cases}
$$

and it also holds that:

$$
\left.u\left(\xi_{ \pm}(s), s\right)=v_{ \pm}(s) \text { for } \xi_{ \pm}(s) \in\right]-L, L[.
$$


In 35 , the notion of BV-entropy solution $v$ to the steady state equation associated to (27) in the case of constant boundary data $u_{L / R}(t) \equiv u_{L / R}$ has been defined:

$$
\left\{\begin{array}{l}
\left.\partial_{x} f(v)=k(x) g(v) \text { with } x \in\right]-L, L[ \\
v(-L)=u_{L} ; v(L)=u_{R}
\end{array}\right.
$$

The proposed definition is able to handle shock-solutions for (33). In this work, we shall restrict ourselves to essentially smooth solutions:

Proposition 3 ([35]). In any of the following cases, there exists a unique $v \in$ $B V(-L, L)$ entropy solution of 33 :

- expansion: $u_{L} \leq 0 \leq u_{R}, v(x) \equiv 0$,

- left-wind: $u_{L}>0$ and $u_{R}>0, v \in C^{1}([-L, L[)$,

- right-wind: $u_{L}<0$ and $\left.\left.u_{R}<0, v \in C^{1}(]-L, L\right]\right)$,

together with the boundary data: $v(-L) \in I^{-}\left(u_{L}\right), v(L) \in I^{+}\left(u_{R}\right)$.

Of course, these entropy solutions may be discontinuous on the lines $x= \pm L$. Their main interest lies in the following result.

Proposition 4 ([35]). Under the hypotheses (28]), (29], there exists a time $T^{*} \in$ $\mathbb{R}^{+}$depending only on $f, g, L$ such that the entropy solution of (27) satisfies:

$$
t \geq T^{*} \Rightarrow u(x, t)=v(x) \text { for } x \in[-L, L] .
$$

In other words, the entropy solution of (27) becomes one-dimensional within a finite time. Under the additional assumption $f^{\prime} \neq 0$, we can give a refined estimate $T^{*}=O(L)$ which is but a direct consequence of steps (i), (ii) in Lemma 4.3, [35].

3.3. Introduction of a nonconservative equation. We plan now to study the behavior of entropy solutions, 31, to the following sequence of balance laws:

$$
\left\{\begin{array}{l}
\partial_{t} u^{\varepsilon}+\partial_{x} f\left(u^{\varepsilon}\right)=g\left(u^{\varepsilon}\right) \partial_{x} a^{\varepsilon} \\
u^{\varepsilon}(., 0)=u_{0}
\end{array}\right.
$$

where, given a parameter $h>0$, the function $a^{\varepsilon}(x)$ is defined as follows:

$$
a^{\varepsilon}(x)= \begin{cases}K(j h), & x \in\left[j h,\left(j+\frac{1}{2}-\frac{\varepsilon}{2}\right) h[,\right. \\ K\left(\left(j+\frac{1}{2}\right) h\left(1-\frac{1}{\varepsilon}\right)+\frac{x}{\varepsilon}\right), & x \in\left[\left(j+\frac{1}{2}-\frac{\varepsilon}{2}\right) h,\left(j+\frac{1}{2}+\frac{\varepsilon}{2}\right) h[,\right. \\ K((j+1) h), & x \in\left[\left(j+\frac{1}{2}+\frac{\varepsilon}{2}\right) h,(j+1) h[.\right.\end{cases}
$$

Of course, the function $K \in C^{2}(\mathbb{R})$ is an antiderivative of $k$, (26), that is $K^{\prime}(x)=$ $k(x)$. The Kružkov's theory ensures that for each $\varepsilon>0$, there exists a unique entropy solution of (34), 3ut since

$$
a^{\varepsilon \stackrel{\varepsilon \rightarrow 0}{\rightarrow}} a^{h} \stackrel{\text { def }}{=} \sum_{j \in \mathbb{Z}} K(j h) \mathbf{1}_{x \in\left[\left(j-\frac{1}{2}\right) h,\left(j+\frac{1}{2}\right) h[\right.},
$$

the term lying at the right-hand side of (34) becomes ambiguous in the limit $\varepsilon \rightarrow 0$.

Lemma 7. Assume (28), (29) and $u_{0} \in L^{1} \cap B V(\mathbb{R})$ is such that $f^{\prime}\left(u_{0}\right) \geq 0$. Then the sequence $u^{\varepsilon}$ is relatively compact in the strong topology of $L_{\text {loc }}^{1}(\mathbb{R} \times] 0, T[)$ as $\varepsilon \rightarrow 0$. 
Proof. We first recall the function $\phi$ from the very early papers, [23, 22, 17] (see also the standing wave curves (11), (12) in [27]):

$$
\phi^{\prime}(u)=\frac{f^{\prime}(u)}{g(u)} .
$$

We now define a "Riemann invariant" (this terminology will become clear later on):

$$
w(u, a)=\phi^{-1} \circ(\phi(u)-a) .
$$

The main feature is that:

$$
\partial_{u} w(u, a)=\frac{\phi^{\prime}(u)}{\phi^{\prime} \circ w(u, a)} \quad \text { and } \quad \partial_{a} w(u, a)=\frac{-1}{\phi^{\prime} \circ w(u, a)} .
$$

We notice that the points $\bar{u} \in \mathbb{R}_{*}$ for which $g(\bar{u})=0$ are not really singular since 1

$$
g(\bar{u})=0 \Leftrightarrow w(\bar{u}, a)=\bar{u} .
$$

All this to deduce the following: for any $u \in \mathbb{R}$ such that $f^{\prime}(u)>0$ and $a \in \mathbb{R}$, there exist two constants $c, C$ such that:

$$
0<c \leq \partial_{u} w(u, a) \leq C<+\infty .
$$

In particular, the classical theory for (34) ensures that provided (29) holds, [31, 17]:

$$
\left\|u^{\varepsilon}\right\|_{L^{\infty}(\mathbb{R} \times[0, T])} \leq \max \left(U,\left\|u_{0}\right\|_{L^{\infty}(\mathbb{R})}\right) .
$$

Thus we can define another constant as follows:

$$
\bar{C}=\max \left(\frac{g^{\prime}(0)}{f^{\prime \prime}(0)} \sup _{u^{\varepsilon} \neq 0} \frac{\left|g\left(u^{\varepsilon}\right)\right|}{f^{\prime}\left(u^{\varepsilon}\right)}\right) .
$$

Under the prescriptions of Lemma 7, we have $f^{\prime}\left(u^{\varepsilon}\right)>0$ and $a^{\varepsilon} \in B V(\mathbb{R}) \subset L^{\infty}(\mathbb{R})$ and (38) holds for $w\left(u^{\varepsilon}, a^{\varepsilon}\right)$. Plugging (37) into (34), we see that we have, at least in the sense of duality, [5], (see also (5) in [22]):

$$
\partial_{t} w\left(u^{\varepsilon}, a^{\varepsilon}\right)+f^{\prime}\left(u^{\varepsilon}\right) \partial_{x} w\left(u^{\varepsilon}, a^{\varepsilon}\right)=0 .
$$

Using the upper bound in (38), we derive from 400)

$$
T V(w)(., t) \leq T V(w)\left(u_{0}, a^{\varepsilon}\right) \leq C \cdot T V\left(u_{0}\right)+\bar{C} \cdot T V\left(a^{\varepsilon}\right),
$$

which implies that $w\left(u^{\varepsilon}, a^{\varepsilon}\right) \in B V(\mathbb{R} \times[0, T])$ (see the proof of Lemma $\mathbb{1}$ ). Thanks to the lower bound in (38), this entails control on $u^{\varepsilon}$ :

$$
T V\left(u^{\varepsilon}\right)(., t) \leq \frac{1}{c} T V(w)\left(u_{0}, a^{\varepsilon}\right)+\bar{C} \cdot T V\left(a^{\varepsilon}\right) .
$$

Thus we complete the proof using Helly's theorem.

For instance, we select $f(u)=\frac{u^{2}}{2}$ and $g(u)=u(1-u)$ in (34) with $u_{0} \in[0,1]$. This gives $w(u, a)=1-\exp (\ln (1-u)+a)=1-\exp (a)+u \exp (a)$. One can see therefore that $w(1, a)=1$, but $w(0, a)=1-\exp (a) \neq 0$ in general. At this point, we have a problem since $f^{\prime}(0)=g(0)=0$.

Of course, it is always possible to reverse the signs. The point here is to avoid any resonant situation $f^{\prime}\left(u^{\varepsilon}\right)=0$ : we refer to [27, 34] for a study of resonance in the context of balance laws; see also [13, 30].

\footnotetext{
${ }^{1}$ Suppose $u \rightarrow \bar{u}$ in $\mathbb{R}$, then $\left|\phi^{\prime}(u)\right| \rightarrow+\infty$. Since $a \in \mathbb{R}, \phi^{-1} \circ(\phi(u)-a) \rightarrow \phi^{-1} \circ \phi(\bar{u})=\bar{u}$. Conversely, if $w(\bar{u}, a)=\bar{u}$, we apply $\phi$ to both sides: if $a \neq 0$, this means that $|\phi(\bar{u})|$ diverges and $g(\bar{u})=0$ because $f, g$ are smooth.
} 
3.4. The quest for the weak- $\star$ limit. Now we want to shed some light on the ambiguous term emanating from the limit $\varepsilon \rightarrow 0$ of (34). Once again, we will use nonconservative products as defined in [32, 36], that is, as the weak- $\star$ limits of the compact sequences $g\left(u^{\varepsilon}\right) \partial_{x} a^{\varepsilon}$.

The next result is therefore a corner stone of our construction.

Proposition 5. Under the assumptions of Lemma 7, there holds:

$$
g\left(u^{\varepsilon}\right) \partial_{x} a^{\varepsilon \text { weak }-\star \mathcal{M}} \sum_{j \in \mathbb{Z}} h\left(\int_{0}^{1} g(\bar{u})(\xi, t) K^{\prime}((j+\xi) h) . d \xi\right) \delta\left(x-\left(j+\frac{1}{2}\right) h\right),
$$

where $\bar{u}$ satisfies the following differential condition for $\xi \in[0,1]$ :

$$
\partial_{\xi} f(\bar{u})=h g(\bar{u}) K^{\prime}((j+\xi) h)
$$

completed by the initial datum for $t \in[0, T]$ and $x=\left(j+\frac{1}{2}\right) h, j \in \mathbb{Z}$ :

$$
\bar{u}(0, t)=u(x-0, t) .
$$

Proof. Let $\psi$ be a continuous function with compact support in $\mathbb{R} \times \mathbb{R}_{*}^{+}$. The object under the scope is:

$$
\begin{aligned}
\int_{\mathbb{R} \times \mathbb{R}_{*}^{+}} & \left(g\left(u^{\varepsilon}\right) \partial_{x} a^{\varepsilon}(x, t)\right) \psi(x, t) \cdot d x \cdot d t \\
= & \int_{\mathbb{R}_{*}^{+}}\left(\sum_{j \in \mathbb{Z}} \int_{\left(j+\frac{1}{2}-\frac{\varepsilon}{2}\right) h}^{\left(j+\frac{1}{2}+\frac{\varepsilon}{2}\right) h} g\left(u^{\varepsilon}\right) \frac{1}{\varepsilon} K^{\prime}\left(\left(j+\frac{1}{2}\right) h\left(1-\frac{1}{\varepsilon}\right)+\frac{x}{\varepsilon}\right) \psi(x, t) \cdot d x\right) \cdot d t .
\end{aligned}
$$

Since $K^{\prime}$ and $\psi$ have compact supports in $\mathbb{R}$, the summation runs only over a finite number of indices $j \in \mathcal{J} \subset \mathbb{Z}$. Let us pick a $j_{\ell} \in \mathcal{J}, \ell \in \mathbb{N}$. Since $f^{\prime}\left(u^{\varepsilon}\right)>0, u^{\varepsilon}$ and $\tilde{u}^{\varepsilon}$ defined as follows coincide on the $x$-stripe specified below:

$$
\left\{\begin{array}{l}
\partial_{t} \tilde{u}^{\varepsilon}+\partial_{x} f\left(\tilde{u}^{\varepsilon}\right)=g\left(\tilde{u}^{\varepsilon}\right) \partial_{x} a^{\varepsilon} \\
x \in\left[\left(j_{\ell}+\frac{1}{2}-\frac{\varepsilon}{2}\right) h,\left(j_{\ell}+\frac{1}{2}+\frac{\varepsilon}{2}\right) h\right], t>0, \\
\tilde{u}^{\varepsilon}\left(\left(j_{\ell}+\frac{1}{2}-\frac{\varepsilon}{2}\right) h, t\right)=u^{\varepsilon}\left(\left(j_{\ell}+\frac{1}{2}-\frac{\varepsilon}{2}\right) h, t\right), \\
\tilde{u}^{\varepsilon}\left(\left(j_{\ell}+\frac{1}{2}+\frac{\varepsilon}{2}\right) h, t\right) \in I^{+}(0), \\
\tilde{u}^{\varepsilon}(x, 0)=u_{0}(x) .
\end{array}\right.
$$

We perform a change of variables:

$$
[0,1] \ni \xi=\frac{1}{\varepsilon h}\left[x-\left(j_{\ell}+\frac{1}{2}-\frac{\varepsilon}{2}\right) h\right] .
$$

In these new coordinates, the boundary value problem (44) becomes:

$$
\left\{\begin{array}{l}
\varepsilon h \partial_{t} \tilde{u}^{\varepsilon}+\partial_{\xi} f\left(\tilde{u}^{\varepsilon}\right)=h g\left(\tilde{u}^{\varepsilon}\right) K^{\prime}\left(\left(j_{\ell}+\xi\right) h\right), \\
\xi \in[0,1], t>0, \\
\tilde{u}^{\varepsilon}(\xi=0, t)=u^{\varepsilon}\left(\left(j_{\ell}+\frac{1}{2}-\frac{\varepsilon}{2}\right) h, t\right), \\
\tilde{u}^{\varepsilon}(\xi=1, t) \in I^{+}(0), \\
\tilde{u}^{\varepsilon}(\xi, t=0)=u_{0}\left(\varepsilon h \xi+\left(j_{\ell}+\frac{1}{2}-\frac{\varepsilon}{2}\right) h\right) .
\end{array}\right.
$$


We plan to follow the ideas of [11, 35] to study:

$$
\begin{aligned}
\int_{\mathbb{R}_{*}^{+}} & \int_{\left(j_{\ell}+\frac{1}{2}-\frac{\varepsilon}{2}\right) h}^{\left(j_{\ell}+\frac{1}{2}+\frac{\varepsilon}{2}\right) h} g\left(u^{\varepsilon}\right)(x, t) \partial_{x} a^{\varepsilon}(x) \psi(x, t) . d x \cdot d t \\
& =\int_{\mathbb{R}_{*}^{+}} h \int_{0}^{1} g\left(\tilde{u}^{\varepsilon}\right)(\xi, t) K^{\prime}\left(\left(j_{\ell}+\xi\right) h\right) \psi\left(\varepsilon h \xi+\left(j_{\ell}+\frac{1}{2}-\frac{\varepsilon}{2}\right) h, t\right) . d \xi . d t .
\end{aligned}
$$

Let us first assume that $\tilde{u}^{\varepsilon}$, the entropy solution of (45), is smooth inside the support of the test function. Thus one can express it by means of the characteristics method:

$$
\dot{\xi}_{\tau_{0}}(t)=\frac{1}{\varepsilon h} f^{\prime}(v)(t), \quad \dot{v}(t)=\frac{1}{\varepsilon} K^{\prime}\left(\left(j_{\ell}+\xi_{\tau_{0}}(t)\right) h\right) g(v)(t),
$$

with $\xi_{\tau_{0}}\left(\tau_{0}\right)=0$ and $v\left(\tau_{0}\right)=u^{\varepsilon}\left(\left(j_{\ell}+\frac{1}{2}-\frac{\varepsilon}{2}\right) h, \tau_{0}\right)$. Since $f^{\prime}\left(\tilde{u}^{\varepsilon}\right)>0$, the entropy solution $\tilde{u}^{\varepsilon}$ does not depend on $u_{0}$ beyond a time $O(\varepsilon)$; therefore, we always consider that the backward characteristics trace up to the left boundary of the stripe. Since $\tilde{u}^{\varepsilon}(\xi, t)=v(t)$ along $t \mapsto \xi_{\tau_{0}}(t)$, we get that:

$$
\partial_{\xi} f\left(\tilde{u}^{\varepsilon}\right)(\xi, t)=f^{\prime}(v) \frac{d v}{d t}\left(\dot{\xi}_{\tau_{0}}\right)^{-1}(t)=h K^{\prime}\left(\left(j_{\ell}+\xi_{\tau_{0}}(t)\right) h\right) g\left(\tilde{u}^{\varepsilon}\right)(\xi, t),
$$

together with $\tilde{u}^{\varepsilon}\left(\xi=0, \tau_{0}\right)=u^{\varepsilon}\left(\left(j_{\ell}+\frac{1}{2}-\frac{\varepsilon}{2}\right) h, \tau_{0}\right)$. For any $\varepsilon>0, \xi_{\tau_{0}}$ is a genuine characteristic and therefore realizes a diffeomorphism $\left[\tau_{0}, t\right] \rightarrow[0, \xi]$. We denote by $\tau$ its inverse mapping satisfying $\tau(\xi)=t$ and we compute:

$$
\frac{d \tau}{d \xi}(\xi)=\frac{\varepsilon h}{f^{\prime}(v)(\tau(\xi))} \stackrel{\varepsilon \rightarrow 0}{\rightarrow} 0
$$

So, the proposition holds for smooth $\tilde{u}^{\varepsilon}$. Now, if $\xi, t$ is a shock location, we use the results of Proposition 2. We apply the same ideas to $\xi_{ \pm}$, the maximal/minimal backward characteristics tracing back to the left boundary at times $\left(\tau_{0}\right)_{ \pm}$. We observe that $\left|\left(\tau_{0}\right)_{+}-\left(\tau_{0}\right)_{-}\right| \leq O(\varepsilon)$ and that $\left|\xi_{+}(s)-\xi_{-}(s)\right| \rightarrow 0$ as $\varepsilon \rightarrow 0$ for $s \in\left[\max \left(\tau_{0}\right)_{ \pm}, t\right]$. This completes the proof.

In some particular cases, for instance if $\operatorname{supp}(k) \subset[j h,(j+1) h]$ for a $j \in \mathbb{Z}$ and $u_{0} \equiv C \in \mathbb{R}^{+}$, Proposition 4 allows one to conclude the proof immediately.

We can reformulate this result using the families of paths as introduced in [12]. This can be stated as follows:

$$
\begin{aligned}
& g\left(u^{\varepsilon}\right) \partial_{x} a^{\varepsilon \text { weak-^ }} \stackrel{\mathcal{M}}{\longrightarrow} \sum_{j \in \mathbb{Z}} h\left[g(u) \partial_{x} a^{h}\right]_{\Phi} \delta\left(x-\left(j+\frac{1}{2}\right) h\right), \\
& {[0,1] \ni s \mapsto \Phi\left(s ;\left(\begin{array}{c}
u \\
a^{h}
\end{array}\right)(x-0),\left(\begin{array}{c}
u \\
a^{h}
\end{array}\right)(x+0)\right)=\left(\begin{array}{c}
\bar{u}(s h) \\
K((j+s) h)
\end{array}\right) .}
\end{aligned}
$$

One can notice a big difference between Proposition[5 and Theorem 2 in [19]: in the former work, the family of paths $\Phi$ was chosen in advance in order to give some correct generalized jump relations at the points where $a^{h}$ was discontinuous. In this new framework, the meaning of the nonconservative product is directly given by the weak- $\star$ limit of some sequences $u^{\varepsilon}, a^{\varepsilon}$ under the constraint of satisfying the equation (34), which means that it is not defined a priori and for any arbitrary choice of $u$ and $a^{h}$. This is somehow a uniqueness result concerning the choice of the family of paths used in the numerical algorithms proposed in [19, 20, 22]. 
An alternative formulation of (34) also used in, e.g., [27, 19] is as follows:

$$
\left\{\begin{array}{l}
\partial_{t} u+\partial_{x} f(u)-g(u) \partial_{x} a=0 \\
\partial_{t} a=0
\end{array}\right.
$$

This "system" is not ambiguous once the initial datum for $a$ is Lipschitz continuous. An easy computation shows that it is nonstrictly hyperbolic with eigenvalues $\Lambda(u)=$ $\left\{0, f^{\prime}(u)\right\}$. Its eigenvectors are:

$$
\vec{R}_{0}=\left(\begin{array}{c}
g(u) \\
f^{\prime}(u)
\end{array}\right), \vec{R}_{f^{\prime}}=\left(\begin{array}{l}
1 \\
0
\end{array}\right) .
$$

Thus, one can check that $w(u, a)$, (37), is a Riemann invariant and that (47) belongs to the Temple class (in the sense that wave curves are Riemann invariant level sets), [38, once (41), (42), (431) rule the discontinuity points of $a$ through some generalized jump relations. Our BV-bound on $w\left(u^{\varepsilon}, a^{\varepsilon}\right)$ (see the proof of Lemma 7) was somehow predictable from this perspective.

A "conservative version" of (47),

$$
\left\{\begin{array}{l}
\partial_{t} u+\partial_{x} f(u, a)=0 \\
\partial_{t} a=0
\end{array}\right.
$$

has been studied extensively in [2] in strictly hyperbolic case, i.e., under the assumption that $\partial_{u} f(u, a)>0$ in the context of $L^{1} \cap L^{\infty}$ functions. The general situation including resonant regimes has been tackled by the authors of [30].

3.5. A uniqueness result "à la Kružkov". Now we want to prove uniqueness for the limit equation:

$$
\left\{\begin{array}{l}
\partial_{t} u+\partial_{x} f(u)=\sum_{j \in \mathbb{Z}}[K((j+1) h)-K(j h)] g(u) \delta\left(x-\left(j+\frac{1}{2}\right) h\right), \\
u(., 0)=u_{0} \in L^{1} \cap B V(\mathbb{R}),
\end{array}\right.
$$

where the measure-valued source term has to be understood by means of Proposition 5 Following the classical works of Lax, we call entropy solution of (49) a function belonging to $B V(\mathbb{R} \times[0, T])$ such that, for any entropy pair $(\eta, q)$ with $\eta \in C^{2}(\mathbb{R})$ strictly convex and $q^{\prime}=\eta^{\prime} f^{\prime}$, it holds that:

$$
\partial_{t} \eta(u)+\partial_{x} q(u) \leq \eta^{\prime}(u) g(u) \partial_{x} a^{h}
$$

in the sense of distributions on $\mathbb{R} \times \mathbb{R}_{*}^{+}$. The right-hand side of (50) has the following meaning:

$$
\begin{aligned}
\eta^{\prime}(u) & g(u) \partial_{x} a^{h} \\
\quad= & \sum_{j \in \mathbb{Z}} h\left(\int_{0}^{1} \eta^{\prime}(\bar{u}) g(\bar{u})(\xi, t) K^{\prime}((j+\xi) h) \cdot d \xi\right) \delta\left(x-\left(j+\frac{1}{2}\right) h\right),
\end{aligned}
$$

with $\bar{u}$ defined as in (42), (43). The first step is to introduce the weak entropies of Kružkov.

Lemma 8. An entropy solution of (49) satisfies the following for every $l \in \mathbb{R}$ :

$$
\partial_{t}|u-l|+\partial_{x}\{\operatorname{sgn}(u-l)(f(u)-f(l))\} \leq \operatorname{sgn}(u-l) g(u) \partial_{x} a^{h}
$$

in the sense of distributions on $\mathbb{R} \times \mathbb{R}_{*}^{+}$. 
Proof. Let $N \in C^{2}(\mathbb{R})$ be such that:

$$
N^{\prime \prime} \geq 0 \text { and } N(x)=|x| \text { for }|x| \geq 1 .
$$

We then define a sequence $N^{\varepsilon}(x)=\varepsilon N\left(\frac{x-l}{\varepsilon}\right)$ converging in $C^{0}(\mathbb{R})$ towards $x \mapsto$ $|x-l|$ as $\varepsilon \rightarrow 0$. Therefore, (50) holds for each $N^{\varepsilon}, \varepsilon>0$. The point to be checked is the behavior of the measure source term; because of its definition (151), we have for every $j \in \mathbb{Z}$ :

$$
\int_{0}^{1}\left(N^{\varepsilon}\right)^{\prime}(\bar{u}) g(\bar{u})(\xi, t) K^{\prime}((j+\xi) h) \cdot d \xi \leq\left\|\left(N^{\varepsilon}\right)^{\prime}(u) g(u)\right\|_{L^{\infty}\left(\mathbb{R} \times \mathbb{R}^{+}\right)} \cdot\|k\|_{L^{1}(\mathbb{R})} .
$$

Thanks to this bound, we get convergence of the weights of the Dirac masses by means of Lebesgue's dominated convergence theorem. The treatment of the lefthand side of (501) is standard. This way, we also get the meaning of the right-hand side of (52).

A particularly interesting situation from a computational viewpoint is the Riemann problem for (49) corresponding to initial data of the form:

$$
u(x, 0)=\left\{\begin{array}{lll}
u_{L} & \text { if } \quad x<0 \\
u_{R} & \text { if } \quad x>0 .
\end{array}\right.
$$

Its entropy solution is made of two elementary waves; we refer to [19, 23] for more details in this direction. The general results of [12, 32, 36] guarantee uniqueness for these Riemann problems, but the next lemma establishes uniqueness for the Cauchy problem (49) under some restriction on the right-hand side.

Lemma 9. Assume $g^{\prime} \leq 0$ and consider two initial data $u_{0}, v_{0}$ for (49): the corresponding entropy solutions $u, v$ satisfy for all $(t, R) \in[0, T] \times \mathbb{R}^{+}$:

$$
\int_{|x| \leq R}|u(s, t)-v(s, t)| d s \leq \int_{|x| \leq R+M t}\left|u_{0}(s)-v_{0}(s)\right| d s,
$$

where $M=\sup \left|f^{\prime}(u)\right|$.

Proof. We select special positive test functions in $\mathcal{D}\left(\left(\mathbb{R} \times \mathbb{R}_{*}^{+}\right)^{2}\right)$, namely:

$$
\Psi(x, t, y, s)=\psi(x, t) \zeta(x-y) \zeta(t-s),
$$

with $\psi \in \mathcal{D}\left(\mathbb{R} \times \mathbb{R}_{*}^{+}\right)$and $\zeta$ is a $C^{\infty}$ approximation of the Dirac mass. We proceed by approximation: let $\left(u^{\varepsilon}, v^{\varepsilon}\right) \in B V(\mathbb{R} \times[0, T])$ be entropy solutions of (34) associated to the initial data $\left(u_{0}, v_{0}\right) \in L^{1} \cap B V(\mathbb{R})$.

We write down the classical entropy inequalities for $u^{\varepsilon}(x, t), v^{\varepsilon}(y, s)$; we add and test on $\left(\mathbb{R} \times \mathbb{R}_{*}^{+}\right)^{2}$ with $\Psi \geq 0$ :

$$
\begin{aligned}
& \int_{\left(\mathbb{R} \times \mathbb{R}_{*}^{+}\right)^{2}}\left\{\left|u^{\varepsilon}(x, t)-v^{\varepsilon}(y, s)\right| \partial_{t} \psi(x, t)\right. \\
&+\operatorname{sgn}\left(u^{\varepsilon}(x, t)-v^{\varepsilon}(y, s)\right)\left(f\left(u^{\varepsilon}\right)(x, t)-\right.\left.f\left(v^{\varepsilon}\right)(y, s)\right) \partial_{x} \psi(x, t) \\
&\left.+\operatorname{sgn}\left(u^{\varepsilon}(x, t)-v^{\varepsilon}(y, s)\right)\left(g\left(u^{\varepsilon}\right)(x, t) \partial_{x} a^{\varepsilon}(x)-g\left(v^{\varepsilon}\right)(y, s) \partial_{y} a^{\varepsilon}(y)\right) \psi(x, t)\right\} \\
& \times \zeta(x-y) \zeta(t-s) . d x . d y . d s . d t \geq 0 .
\end{aligned}
$$

We just split the part devoted to the source term:

$$
\begin{aligned}
& g\left(u^{\varepsilon}\right)(x, t) \partial_{x} a^{\varepsilon}(x)-g\left(v^{\varepsilon}\right)(y, s) \partial_{y} a^{\varepsilon}(y) \\
& \quad \leq\left[g\left(u^{\varepsilon}\right)(x, t)-g\left(v^{\varepsilon}\right)(y, s)\right] \partial_{x} a^{\varepsilon}(x)+\frac{1}{\varepsilon}\left\|g\left(v^{\varepsilon}\right)\right\|_{L^{\infty}}\left|K^{\prime}(x)-K^{\prime}(y)\right| .
\end{aligned}
$$


At this level, the standard theory of Kružkov permits us to let $\zeta$ concentrate to the Dirac measure $\delta$ thanks to the BV regularity of $u$ and $v$. We now have:

$$
\begin{aligned}
\int_{\mathbb{R} \times \mathbb{R}_{*}^{+}}\{\mid & u^{\varepsilon}(x, t)-v^{\varepsilon}(x, t) \mid \partial_{t} \psi(x, t) \\
& +\operatorname{sgn}\left(u^{\varepsilon}(x, t)-v^{\varepsilon}(x, t)\right)\left(f\left(u^{\varepsilon}\right)(x, t)-f\left(v^{\varepsilon}\right)(x, t)\right) \partial_{x} \psi(x, t) \\
& \left.+\operatorname{sgn}\left(u^{\varepsilon}-v^{\varepsilon}\right)\left(g\left(u^{\varepsilon}\right)-g\left(v^{\varepsilon}\right)\right) \partial_{x} a^{\varepsilon}(x) \psi(x, t)\right\} . d x . d t \geq 0 .
\end{aligned}
$$

Taking into account the signs of $g^{\prime}$ and $\partial_{x} a^{\varepsilon}$, we invoke the following inequality:

$$
\int_{\mathbb{R} \times \mathbb{R}_{*}^{+}} \operatorname{sgn}\left(u^{\varepsilon}-v^{\varepsilon}\right)\left(g\left(u^{\varepsilon}\right)-g\left(v^{\varepsilon}\right)\right) \partial_{x} a^{\varepsilon}(x) \psi(x, t) . d x . d t \leq 0 .
$$

The entropy inequality becomes:

$$
\int_{\mathbb{R} \times \mathbb{R}_{*}^{+}}\left\{|u(x, t)-v(x, t)| \partial_{t} \psi(x, t)+\operatorname{sgn}(u-v)(f(u)-f(v)) \partial_{x} \psi(x, t)\right\} . d x . d t \geq 0 .
$$

Now, it remains to select $\psi$ using regularized Heaviside functions as in, e.g., [31, 6 . 29]; the fluxes and the remaining terms cancel. We finally derive:

$$
\int_{\mathbb{R} \times \mathbb{R}_{*}^{+}}|u(x, t)-v(x, t)| \partial_{t} \psi(x, t) . d x \cdot d t \geq 0 .
$$

We close this section with the construction of $\mathbf{S}$, the solution operator for (49) mapping $L^{1} \cap B V(\mathbb{R})$ into itself. We refer again to [2] for the construction of the solution operator for (48) in the strictly hyperbolic case which turns out to be a semigroup whose domain is $L^{1} \cap L^{\infty}(\mathbb{R})$. Here, the $\mathrm{BV}$ regularity is needed at least to define the nonconservative products.

Proposition 6. There exists a unique "nonconservative contraction semigroup" $\mathbf{S}$ whose domain is $\mathbf{D}=L^{1} \cap B V(\mathbb{R})$ and such that every trajectory coincides with the entropy solution to (49), (28), (29), $g^{\prime} \leq 0$. More precisely, for every $s \geq 0, t \geq 0$, it holds that

$$
\mathbf{S}(t)(u(., s))=u(., s+t) .
$$

Proof. Since we avoided any explicit dependance in the time variable, we have:

$$
\mathbf{S}(0)=\operatorname{Id} \text { and } \mathbf{S}(t) \circ \mathbf{S}(s)=\mathbf{S}(s) \circ \mathbf{S}(t)
$$

whatever the initial data in $\mathbf{D}$ satisfying the requirements of Lemma 7 .

3.6. Convergence of the well-balanced scheme. The final step is the study of the numerical scheme built on this approach. As usual, we define a cartesian computational grid determined by the parameters $h$ and $\Delta t$ standing, respectively, for the space and the time steps. We plan to construct a piecewise constant approximation of the entropy solution of (26) by means of a Godunov scheme using as building blocks Riemann problems for (49). More precisely, an initial datum for (26) being given, we introduce a projector onto piecewise constant functions:

$$
\begin{aligned}
\mathcal{P}^{h}: L^{1} \cap B V(\mathbb{R}) & \rightarrow L^{1} \cap B V(\mathbb{R}), \\
\varphi & \mapsto\left(\varphi_{j}\right)_{j \in \mathbb{Z}} \stackrel{\text { def }}{=}\left(\frac{1}{h} \int_{\left(j-\frac{1}{2}\right) h}^{\left(j+\frac{1}{2}\right) h} \varphi(x) \cdot d x\right)_{j \in \mathbb{Z}} .
\end{aligned}
$$


We denote $C_{j}=\left[\left(j-\frac{1}{2}\right) h,\left(j+\frac{1}{2}\right) h[\right.$. The sequence of values is updated by solving Riemann problems for (49) at each endpoint of $C_{j}, j \in \mathbb{Z}$, and averaging these elementary solutions. In a compact form, the scheme reads:

$$
u^{h}(., t)=\mathbf{S}(n \Delta t-t) \circ\left[\mathcal{P}^{h} \circ \mathbf{S}(\Delta t)\right]^{n}\left(\tilde{u}_{0}\right),
$$

where $\tilde{u}_{0}=\left(u_{0}(j h) \mathbf{1}_{C_{j}}\right)_{j \in \mathbb{Z}}, \mathbf{S}$ is the semi-group introduced in Proposition 6 and $n \in \mathbb{N}$ is the integer part of $t / \Delta t$. We now want to prove stability for this numerical scheme under appropriate restrictions on $\Delta t$ and $h$. Of course, we already assumed the conditions required for the use of the "nonconservative semi-group" $\mathbf{S}$ which means essentially that no resonant regimes should appear.

Lemma 10. Under the restrictions of Lemma 7 , the sequence of piecewise constant approximations $u^{h}\left(\right.$ (54) is relatively compact in $L_{\mathrm{loc}}^{1}(\mathbb{R} \times] 0, T[)$ as $h \rightarrow 0$ under the CFL condition $\sup \left|f^{\prime}(u)\right| \frac{\Delta t}{h} \leq 1$.

Proof. We assume that $f^{\prime}\left(u_{0}\right) \geq 0$; the opposite case is treated the same way.

- We begin by studying the generalized jump condition induced by (42), (43). Since $f^{\prime} \neq 0$ and thanks to (28), (29), (39), we have a maximum principle for $\bar{u}$, namely:

$$
\|\bar{u}\|_{\left.\left.L_{\text {loc }}^{\infty}([0,1] \times] 0, T\right]\right)} \leq \max \left(U,\left\|u_{0}\right\|_{L^{\infty}(\mathbb{R})}\right) .
$$

Our Godunov scheme reads:

$$
\left\{\begin{array}{l}
u^{h}(x, t)=u_{j}^{n} \text { for } x \in C_{j}, t=n \Delta t, \\
u_{j}^{n+1}=u_{j}^{n}-\frac{\Delta t}{h}\left\{f\left(u_{j}^{n}\right)-f\left(u_{j-\frac{1}{2}}^{n}\right)\right\},
\end{array}\right.
$$

together with:

$$
u_{j-\frac{1}{2}}^{n}=\bar{u}(1, n \Delta t) ; \bar{u}(0, n \Delta t)=u_{j-1}^{n} .
$$

At this point, one can use the mean-value theorem to see that the maximum principle holds for $u^{h}$ as a consequence of the CFL restriction:

$$
\left\|u^{h}\right\|_{\left.\left.L_{\text {loc }}^{\infty}(\mathbb{R} \times] 0, T\right]\right)} \leq \max \left(U,\left\|u_{0}\right\|_{L^{\infty}(\mathbb{R})}\right) .
$$

- We now study the spatial total-variation of the scheme (55), (56):

$$
\begin{aligned}
u_{j+1}^{n+1}-u_{j}^{n+1}=u_{j+1}^{n}-u_{j}^{n} & -\frac{\Delta t}{h}\left\{f\left(u_{j+1}^{n}\right)-f\left(u_{j}^{n}\right)\right\} \\
& +\frac{\Delta t}{h}\left\{f\left(u_{j}^{n}\right)-f\left(u_{j-1}^{n}\right)\right\} \\
& +\frac{\Delta t}{h}\left\{f\left(u_{j+\frac{1}{2}}^{n}\right)-f\left(u_{j}^{n}\right)\right\} \\
& -\frac{\Delta t}{h}\left\{f\left(u_{j-\frac{1}{2}}^{n}\right)-f\left(u_{j-1}^{n}\right)\right\} .
\end{aligned}
$$

By definition of the generalized jump relations, see, e.g., [12, 19], we have:

$$
f\left(u_{j+\frac{1}{2}}^{n}\right)-f\left(u_{j}^{n}\right)=h \int_{0}^{1} g(\bar{u})(\xi, n \Delta t) K^{\prime}((j+\xi) h) . d \xi .
$$

It is therefore convenient to introduce a new piecewise $C^{1}$ function $w$ by gluing together all the microscopic profiles $\bar{u}$. It reads:

$$
\forall(j, n) \in \mathbb{Z} \times \mathbb{N},\left\{\begin{array}{l}
w((j+\xi) h, t)=\bar{u}(\xi, t) ; \bar{u}(0, t)=u_{j}^{n}, \\
\xi \in[0,1[, t \in[n \Delta t,(n+1) \Delta t[.
\end{array}\right.
$$


Thus, the last two terms in (57) boil down to:

$$
\begin{aligned}
\Delta t \int_{0}^{1} & {\left[g(w)((j+\xi) h, n \Delta t) K^{\prime}((j+\xi) h)\right.} \\
& \left.-g(w)((j-1+\xi) h, n \Delta t) K^{\prime}((j-1+\xi) h)\right] . d \xi \\
\leq & \Delta t \underbrace{\left\|g^{\prime}\left(u^{h}\right)\right\|_{L^{\infty}}\left\|K^{\prime}\right\|_{C^{0}} \exp \left(h\left\|K^{\prime}\right\|_{C^{0}} \operatorname{Lip}\left(g / f^{\prime}\right)\right)}_{\tilde{N}}\left|u_{j}^{n}-u_{j-1}^{n}\right| \\
& +\Delta t\left\|g\left(u^{h}\right)\right\|_{L^{\infty}} \underbrace{\int_{0}^{1}\left|K^{\prime}((j+\xi) h)-K^{\prime}((j-1+\xi) h)\right| . d \xi}_{T V(k)} .
\end{aligned}
$$

This last bound is a consequence of the classical theory of ordinary differential equations. We can use the mean-value theorem and sum over $j \in \mathbb{Z}$ to get:

$$
\begin{aligned}
T V\left(u^{h}(.,(n+1) \Delta t)\right) \leq & \sum_{j \in \mathbb{Z}}\left(1-\frac{\Delta t}{h} f^{\prime}\left(\gamma_{j+\frac{1}{2}}^{n}\right)\right)\left|u_{j+1}^{n}-u_{j}^{n}\right| \\
& +\left(\frac{\Delta t}{h} f^{\prime}\left(\gamma_{j-\frac{1}{2}}^{n}\right)+\Delta t \tilde{N}\right)\left|u_{j}^{n}-u_{j-1}^{n}\right|+\Delta t\left\|g\left(u^{h}\right)\right\|_{L^{\infty}} T V(k) \\
\leq & \exp (n \Delta t \tilde{N})\left\{T V\left(u_{0}\right)+(1-\exp (-n \Delta t \tilde{N}))\left\|u^{h}\right\|_{L^{\infty}} T V(k)\right\} .
\end{aligned}
$$

- To establish the time-equicontinuity property, we first need to bound $u^{h}$ in $L^{1}(\mathbb{R})$. So, we rewrite (55), (56) as follows:

$$
\begin{aligned}
u_{j}^{n+1}= & u_{j}^{n}-\frac{\Delta t}{h}\left\{f\left(u_{j}^{n}\right)-f\left(u_{j-1}^{n}\right)\right\} \\
& +\Delta t \int_{0}^{1} g(w)((j-1+\xi) h, n \Delta t) K^{\prime}((j-1+\xi) h) . d \xi .
\end{aligned}
$$

It remains to use the mean-value theorem since we know, (28), (29), that $f(0)=g(0)=0$ and to take the moduli:

$$
\begin{aligned}
\left\|u^{h}(.,(n+1) \Delta t)\right\|_{L^{1}(\mathbb{R})} & \leq(1+\Delta t . \tilde{N})\left\|u^{h}(., n \Delta t)\right\|_{L^{1}(\mathbb{R})} \\
& \leq \exp (n \Delta t . \tilde{N})\left\|u_{0}\right\|_{L^{1}(\mathbb{R})} .
\end{aligned}
$$

Now, we just write that:

$$
h\left|u_{j}^{n+1}-u_{j}^{n}\right| \leq \Delta t\left[f^{\prime}\left(\gamma_{j}^{n}\right)\left|u_{j}^{n}-u_{j-1}^{n}\right|+\tilde{N} h\left|u_{j}^{n}\right|\right] .
$$

This leads to:

$$
\begin{aligned}
& \left\|u^{h}(.,(n+1) \Delta t)-u^{h}(., n \Delta t)\right\|_{L^{1}(\mathbb{R})} \\
& \qquad \begin{aligned}
\leq \exp (n \Delta t . \tilde{N}) \Delta t & \left\|f^{\prime}\left(u^{h}\right)\right\|_{L^{\infty}} T V\left(u_{0}\right) \\
& \left.+(1-\exp (-n \Delta t . \tilde{N}))\left\|u^{h}\right\|_{L^{\infty}} T V(k)+\tilde{N}\left\|u_{0}\right\|_{L^{1}(\mathbb{R})}\right] .
\end{aligned}
\end{aligned}
$$

This completes the proof.

The Godunov scheme (55), (56) coincides with the so-called "well-balanced" one studied in [22] although its construction is completely different. Indeed, one can see that both approaches boil down to some kind of split-scheme for which the operator 
splitting is made with respect to the space variable (see, e.g., 16, 39 for related former works).

The nature of the limit of this compact sequence $u^{h}$ can be directly revealed by means of the error estimate theorem given in [17]. However, for the sake of completeness, we state now a self-contained proof.

Theorem 2. Assume (28), (29), $u_{0} \in L^{1} \cap B V(\mathbb{R})$ being such that $f^{\prime}\left(u_{0}\right) \geq 0$ and the CFL restriction

$$
\sup _{|u| \leq \max \left\{\left\|u_{0}\right\|_{L^{\infty}(\mathbb{R})}, U\right\}}\left|f^{\prime}(u)\right| \Delta t \leq h ;
$$

the sequence $u^{h}$ generated by (54), (55), (56) converges strongly in $L_{\mathrm{loc}}^{1}(\mathbb{R} \times] 0, T[)$ as $h \rightarrow 0$ towards Kružkov's entropy solution of the following balance law:

$$
\left\{\begin{array}{l}
\partial_{t} u+\partial_{x} f(u)=k(x) g(u) \\
u(., 0)=u_{0} \in L^{1} \cap B V(\mathbb{R})
\end{array}\right.
$$

where $k \in C^{1}(\mathbb{R})$ is positive with compact support.

Once again, under assumptions avoiding any nonlinear resonance, it is possible to weaken the hypotheses on $u_{0}$ as in, e.g., [22].

Proof. We recall the entropy inequality (50), 51) which is satisfied by the solution of any of the elementary Riemann problems for (49) solved inside the scheme (54). So, we take any positive test function $\psi \in \mathcal{D}\left(\mathbb{R} \times \mathbb{R}_{*}^{+}\right)$:

$$
\begin{aligned}
0 \leq & \sum_{j, n} \int_{n \Delta t}^{(n+1) \Delta t} \int_{j h}^{(j+1) h} \eta\left(u^{h}\right) \partial_{t} \psi+q\left(u^{h}\right) \partial_{x} \psi+\eta^{\prime}\left(u^{h}\right) g\left(u^{h}\right) \cdot \partial_{x} a^{h} \psi \cdot d x . d t \\
& +\sum_{j, n} \int_{j h}^{(j+1) h}\left\{\eta\left(u^{h}\right)(x, n \Delta t)-\eta\left(u^{h}\right)(x, n \Delta t-0)\right\} \psi(x, n \Delta t) . d x .
\end{aligned}
$$

Thanks to Jensen's inequality for convex functions, we can get rid of the last term in (61) since $u^{h}(., n \Delta t)=\mathcal{P}^{h}\left(u^{h}(., n \Delta t-0)\right)$.

- Thanks to regularity assumptions on $\psi$, we see immediately that we can split the last part of the first term in (61) using the function $w$ (58):

$$
\begin{aligned}
\sum_{j, n} & \int_{n \Delta t}^{(n+1) \Delta t} \int_{j h}^{(j+1) h} \eta^{\prime}\left(u^{h}\right) g\left(u^{h}\right) \partial_{x} a^{h} \psi(x, t) \cdot d x \cdot d t \\
= & \sum_{j, n} \int_{n \Delta t}^{(n+1) \Delta t}\left(\int_{j h}^{(j+1) h} \eta^{\prime}(w) g(w)(x, t) K^{\prime}(x) \cdot d x\right) \psi((j+1 / 2) h, t) \cdot d t \\
\leq & \sum_{j, n} \int_{n \Delta t}^{(n+1) \Delta t} \int_{j h}^{(j+1) h} \eta^{\prime}(w) g(w)(x, t) K^{\prime}(x) \psi(x, t) \cdot d x \cdot d t \\
& +h\left\|\eta^{\prime}\left(u^{h}\right) g\left(u^{h}\right)\right\|_{L^{\infty}}\left\|K^{\prime}\right\|_{C^{0}}\left\|\partial_{x} \psi\right\|_{L^{1}} .
\end{aligned}
$$


- Now we have to estimate the $L^{1}$-distance between the piecewise constant function $u^{h}(., n . \Delta t)$ and the microscopic profiles $w(., t)$ for $t \in[n \Delta t,(n+1) \Delta t[$. This is a consequence of the classical ordinary differential equations theory together with the non-resonance assumption:

$$
\begin{aligned}
& \int_{n \Delta t}^{(n+1) \Delta t} \int_{j h}^{(j+1) h} \eta^{\prime}(w) g(w)(x, t) K^{\prime}(x) \psi(x, t) \cdot d x \cdot d t \\
& \quad \leq \int_{n \Delta t}^{(n+1) \Delta t} \int_{j h}^{(j+1) h} \eta^{\prime}\left(u^{h}\right) g\left(u^{h}\right)(j h, n \Delta t) K^{\prime}(x) \psi(x, t) \cdot d x \cdot d t+O(h) \tilde{N}\|\psi\|_{L^{1}} .
\end{aligned}
$$

- We write now that:

$$
\begin{aligned}
& \sum_{j, n} \int_{n \Delta t}^{(n+1) \Delta t} \int_{j h}^{(j+1) h} \eta^{\prime}\left(u^{h}\right) g\left(u^{h}\right)(j h, n \Delta t) K^{\prime}(x) \psi(x, t) \cdot d x \cdot d t \\
& \quad \leq \int_{\mathbb{R} \times \mathbb{R}_{*}^{+}} \eta^{\prime}\left(u^{h}\right) g\left(u^{h}\right)(x, t) K^{\prime}(x) \psi(x, t) \cdot d x \cdot d t \\
& \quad+O(1) \sum_{j, n} \int_{n \Delta t}^{(n+1) \Delta t} \int_{j h}^{(j+1) h}\left|u^{h}(x, t)-u^{h}(j h, n \Delta t)\right| \psi(x, t) \cdot d x . d t .
\end{aligned}
$$

Thanks to Lemma 10, we have:

$$
\left|u^{h}(x, t)-u^{h}(j h, n \Delta t)\right| \leq h \exp (t \tilde{N}) T V\left(u_{0}\right)+\left|u^{h}(j h,(n+1) \Delta t)-u^{h}(j h, n \Delta t)\right|
$$

and we know from (59) that $u^{h}$ is $L^{1}$-equicontinuous with respect to the time variable: $\left\|u^{h}(.,(n+1) \Delta t)-u^{h}(., n \Delta t)\right\|_{L^{1}(\mathbb{R})} \leq O(\Delta t)$.

The last step consists of collecting all our information and using Lebesgue's dominated convergence theorem to derive Kružkov's entropy inequality for (26).

3.7. Numerical results. As an illustrative test-case, we consider the following initial-boundary value problem:

$$
\left\{\begin{array}{l}
\partial_{t} u+\partial_{x}\left(\frac{u^{2}}{2}\right)=k(x) u(1-u), \\
x \in[0,1] \text { and } 0<t \leq 3.5 \\
u(0, t)=0.5 ; u(x, 0)=0
\end{array}\right.
$$

As a direct consequence of the results proven by Mascia and Terracina, 35, the steady-state for (62) is given by:

$$
v(x)=1-\frac{1}{2} \exp \left(-\int_{0}^{x} k(s) \cdot d s\right) .
$$

We compare the accuracy of the numerical stationary curves for the proposed nonconservative scheme (55), (56) and a classical time-splitting algorithm for various values of the space step $h$. We chosed the following "delta-like" function:

$$
k(x)=20 . \exp \left(-\frac{(x-0.5)^{2}}{\varepsilon}\right), \quad \varepsilon=0.01 .
$$

At time $T=3.5$, all the waves are out of the domain $x \in[0,1]$ but the schemes did not have time to stabilize completely. We display in Figures 3 and 4 , respectively the numerical solutions and the $L^{\infty}$-distance between themselves and $v$ (63). 


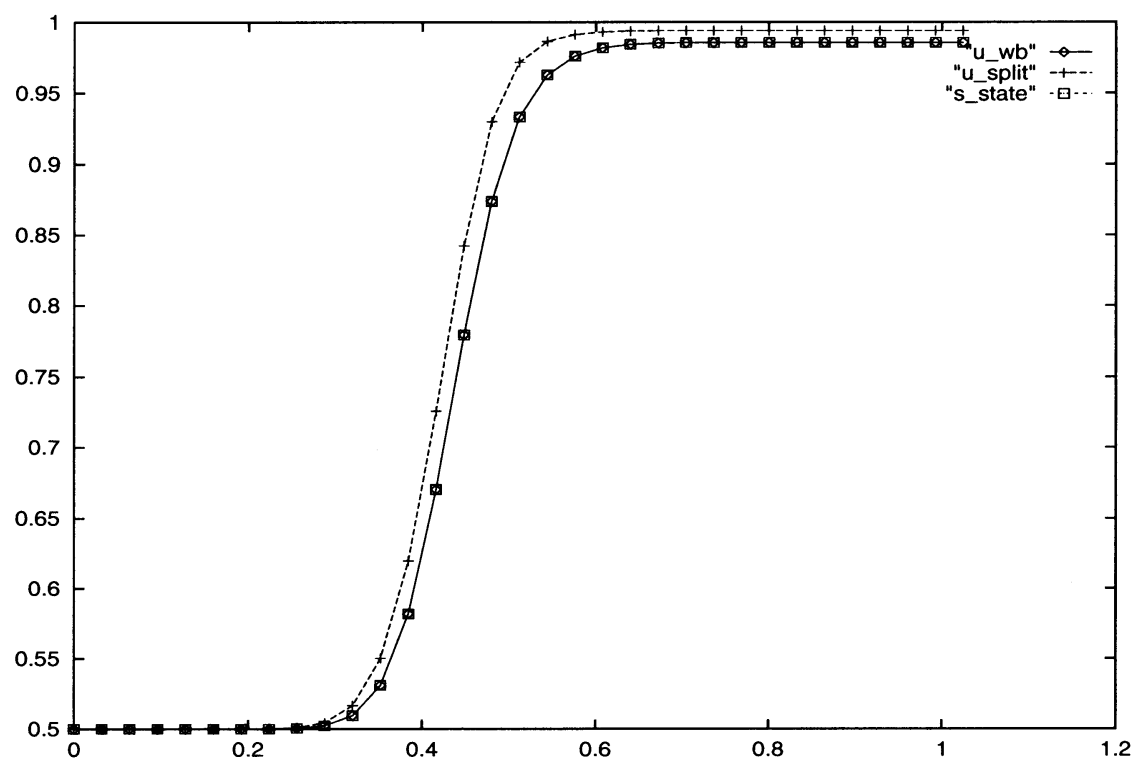

Figure 3. Exact and numerical solutions for (62), (64) at $T=3.5$.

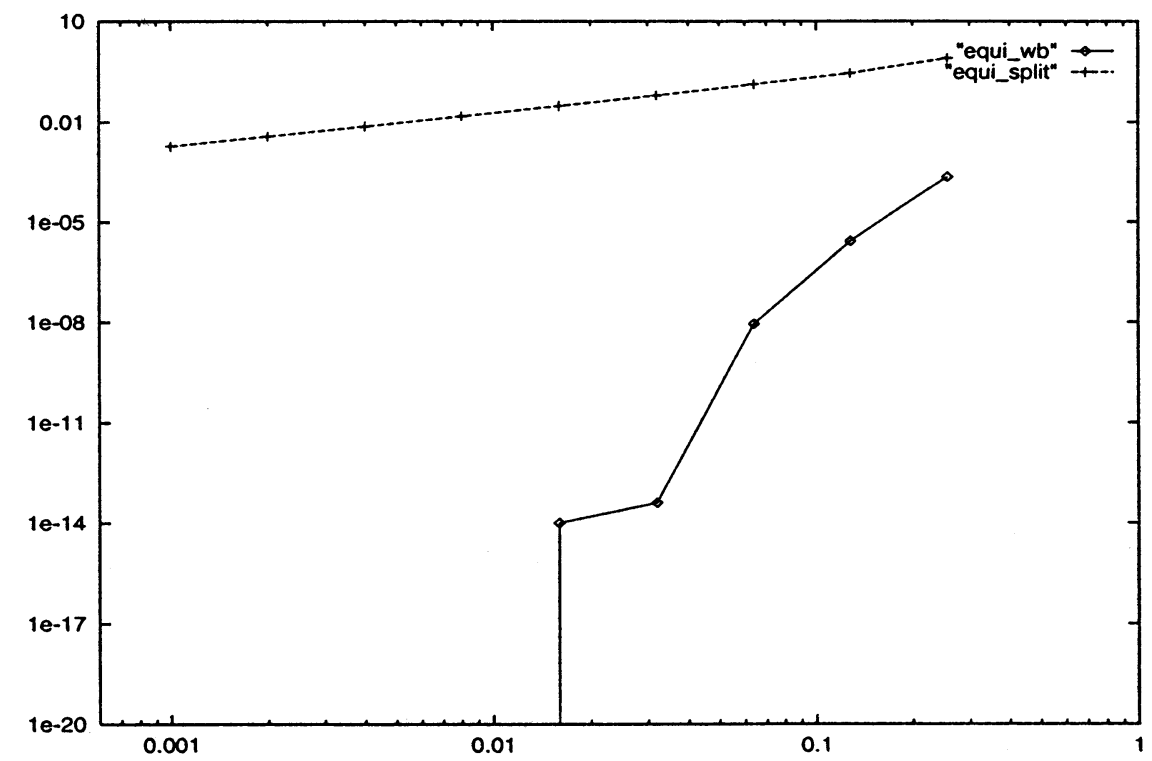

FiguRE 4. Sup-norm decay for $x \mapsto\left|u^{h}(x, T=3.5)-v(x)\right|$ as $h \rightarrow 0$. 
The advantages of this nonconservative approach are clearly noticeable even if theoretical results guarantee the convergence of the classical time-splitting scheme as the grid is refined, see, e.g., 37]. We refer to [8, 19, 23] for other comparisons between well-balanced approaches and more conventional discretizations on more complex and realistic problems.

\section{Conclusion}

We proposed in this paper a new way to construct numerical schemes for nonhomogeneous hyperbolic equations by means of a Godunov scheme involving singular Riemann problems. The ambiguous terms arise naturally as weak limits of strongly compact regularized sequences and somehow contain useful informations about the asymptotic patterns one can expect as a long-time behavior for both exact and numerical solutions. This approach extends formally to systems of balance laws and furnishes high quality numerical results. One perspective is therefore to generalize this scalar theory to more general hyperbolic problems by means, for instance, of a Glimm scheme strategy.

\section{ACKNOWLEDGMENTS}

The author is grateful to Professors A. E. Tzavaras, A. Vasseur and L. Pareschi for their interest in this work which has been partially supported by the European Union TMR project HCL \#ERBFMRXCT960033.

\section{REFERENCES}

[1] D. Aregba-Driollet and R. Natalini, Convergence of relaxation schemes for conservation laws, Appl. Anal., 61 (1996), pp. 163-193. CMP 98:13

[2] P. Baiti And H.K. Jenssen, Well-posedness for a class of $2 \times 2$ conservation laws with $L^{\infty}$ data, J. Diff. Eq. 140 (1997), pp. 161-185. MR 98k:35122

[3] C. Bardos, A.Y. LeRoux, And J.C. Nedelec, First-order quasilinear equations with boundary conditions, Comm. Part. Diff. Equ., 4 (1979), pp. 1017-1034. MR 81b:35052

[4] F. Bouchut, Construction of BGK models with a family of kinetic entropies for a given system of conservation laws, J. Stat. Phys. 95 (1999), pp. 113-170. MR 2000f:82084

[5] F. Bouchut And F. James, One-dimensional transport equations with discontinuous coefficients, Nonlinear Anal. TMA, 32 (1998), pp. 891-933. MR 2000a:35243

[6] F. Bouchut And B. Perthame, Kružkov's inequalities for scalar conservation laws revisited, Trans. Amer. Math. Soc. 350 (1998), pp. 2847-2870. MR 98m:65156

[7] P. A. Burton, Convergence of flux-limiting schemes for hyperbolic conservation laws with source terms, Ph.D. Thesis, Univ. of Reading (1993), available at the following URL: http://www.rdg.ac.uk/AcadDepts/sm/wsm1/theses/pab.html.

[8] P. CARgo AND A.Y. LeRoux, Un schéma équilibre adapté au modèle d'atmosphère avec termes de gravité, C.R. Acad. Sc. Paris Série I, 318 (1994), pp. 73-76. MR 94j:86005

[9] J.J. Cauret, J.F. Colombeau, and A.Y. LeRoux, Discontinuous generalized solutions of nonlinear nonconservative hyperbolic equations, J. Math. Anal. Applic., 139 (1989), pp. 552573. MR 91c:35085

[10] M. CRandall And A. MajDa, Monotone difference approximations for scalar conservation laws, Math. Comp., 34 (1980), pp. 1-21. MR 81b:65079

[11] C. Dafermos, Generalized characteristics and the structure of solutions of hyperbolic conservation laws, Indiana Univ. Math. J. 26 (1977), pp. 1097-1119. MR 56:16151

[12] G. Dal Maso, P.G. LeFloch, and F. Murat, Definition and weak stability of a nonconservative product, J. Math. Pures Appl., 74 (1995), pp. 483-548. MR 97b:46052 
[13] P. Embid, J. Goodman, And A. Majda, Multiple steady states for 1-D transonic flow, SIAM J. Sci. Stat. Comp., 5 (1984), pp. 21-41. MR 86a:76029

[14] B. Engquist And B. SJÖGREen, The convergence rate of finite difference schemes in the presence of shocks, SIAM J. Numer. Anal., 35 (1998), 2464-2485. MR 99k:65080

[15] J. Falcovitz And M. Ben-ARTZI, Recent developments of the GRP method, JSME International J. Series B, 38 (1995), pp. 497-517.

[16] J. Glimm, G. Marshall, and B.J. Plohr, A generalized Riemann problem for quasi one dimensional gas flows, Adv. Appl. Math., 5 (1984), pp. 1-30. MR 85e:76041

[17] L. Gosse, A priori error estimate for a well-balanced scheme designed for inhomogeneous scalar conservation laws, C.R. Acad. Sc. Paris Série I, 327 (1998), pp. 467-472. MR 99h:35126

[18] L. Gosse, Sur la stabilité des approximations implicites des lois de conservation scalaires non-homogènes, C.R. Acad. Sc. Paris Série I, 329 (1999), pp. 79-84. MR 2000d:65152

[19] L. Gosse, A well-balanced scheme using non-conservative products designed for hyperbolic systems of conservation laws with source terms, Math. Mod. Meth. Appl. Sc., 11 (2001), pp. 339-365. CMP 2001:10

[20] L. Gosse, A well-balanced flux splitting scheme designed for hyperbolic systems of conservation laws with source terms, Comp. Math. Applic. 39 (2000), pp. 135-159. CMP 2000:11

[21] L. Gosse And F. James, Numerical approximation of linear one-dimensional conservation equations with discontinuous coefficients, Math. Comp. 69 (2000), pp. 987-1015. MR 2000j:65077

[22] L. Gosse AND A.Y. LeRoux, Un schéma-équilibre adapté aux lois de conservation scalaires non-homogènes, C.R. Acad. Sc. Paris Série I, 323 (1996), pp. 543-546. MR 97i:35112

[23] J. Greenberg And A.Y. LeRoux, A well balanced scheme for the numerical processing of source terms in hyperbolic equations, SIAM J. Numer. Anal., 33 (1996), pp. 1-16. MR 97c:65144

[24] A. Heibig and J.F. Colombeau, Nonconservative products in bounded variation functions, SIAM J. Math. Anal., 23 (1992), pp. 941-949. MR 93f:46058

[25] C. Helzel, R.J. LeVeque, and G. Warnecke, A modified fractional step method for the accurate approximation of detonation waves, SIAM J. Sci. Stat. Comp., 22 (2000), pp. 14891510. MR 2001h:65093

[26] T.Y. Hou And P.G. LeFloch, Why nonconservative schemes converge to wrong solutions: error analysis, Math. Comp., 62 (1993), pp. 497-530. MR 94g:65093

[27] E. IsAaCson AND B. Temple, Convergence of the $2 \times 2$ Godunov method for a general resonant nonlinear balance law, SIAM J. Appl. Math., 55 (1995), pp. 625-640. MR 96c:65146

[28] S. Jin AND Z. XIN, The relaxing schemes for systems of conservation laws in arbitrary space dimensions, Comm. Pure Appl. Math., 48 (1995), pp. 235-276. MR 96c:65134

[29] M.A. Katsoulakis And A.E. Tzavaras, Contractive relaxation systems and the scalar multidimensional conservation law, Comm. Part. Diff. Eqns., 22 (1997), pp. 195-233. MR 97m:35168

[30] R.A. Klausen and N.H. Risebro, Stability of conservation laws with discontinuous coeffcients, J. Diff. Eq. 157 (1999), pp. 41-60. MR 2000m:35124

[31] S.N. KRUŽKov, First order quasilinear equations in several independant space variables, Math. USSR Sbornik, 10 (1970), pp. 217-243. MR 42:2159

[32] P.G. LeFloch and A.E. Tzavaras, Representation of weak limits and definition of nonconservative products, SIAM J. Math. Anal. 30 (1999), pp. 1309-1342. MR 2001e:35113

[33] R.J. LeVeque and H.C. Yee, A study of numerical methods for hyperbolic equations with stiff source terms, J. Comp. Phys., 86 (1990), pp. 187-210. MR 90k:76009

[34] T. P. LiU, Nonlinear resonance for quasilinear hyperbolic equation, J. Math. Phys., 28 (1987), 2593-2602. MR 88k:35122

[35] C. MAscia And A. Terracina, Long-time behavior for conservation laws with source in a bounded domain, J. Diff Eq. 159 (1999), pp. 485-514. MR 2001b:35206

[36] J.P. RaYmond, A new definition of nonconservative products and weak stability results, Boll. Un. Mat. Ital. B (7) 10, (1996), pp. 681-699. MR 97h:46062

[37] T. Tang And Z.H. Teng, Error bounds for fractional step methods for conservation laws with source terms, SIAM J. Numer. Anal. 32 (1995), pp. 110-127. MR 95m:65155

[38] B. Temple, Systems of conservation laws with invariant submanifolds, Trans. Amer. Math. Soc., 280 (1983), pp. 781-795. MR 84m:35080 
[39] B. VANLEER, On the relation between the upwind differencing schemes of Engquist-Osher, Godunov and Roe, SIAM J. Sci. Stat. Comp., 5 (1984), pp. 1-20. MR 86a:65085

[40] A. VASSEur, Kinetic semi-discretization of scalar conservation laws and convergence by using averaging lemmas, SIAM J. Numer. Anal. 36 (1999), pp. 465-474. MR 99m:65174]

[41] A.I. Vol'Pert, The space BV and quasilinear equations, Math. USSR Sbornik, 2 (1967), pp. 225-267. MR 35:7172

Dipartimento di Matematica Pura e Applicata, Università degli Studi di L'Aquila, Via Vetoio, Località Coppito, 67100 L'Aquila, Italy

E-mail address: laurent@teddybear.univaq.it 